$\begin{array}{ll}\text { Italique } & \text { Italique } \\ \text { Poésie italienne de la Renaissance }\end{array}$

XVI | 2013

Varia

\title{
«L'erudita bottega di messer Claudio». Nuovi testi per il Reame della Virtù (Roma 1538)
}

\section{Enrico Garavelli}

\section{(C) OpenEdition}

Journals

\section{Edizione digitale}

URL: http://journals.openedition.org/italique/372

DOI: 10.4000/italique.372

ISSN: 1663-4438

\section{Editore}

Librairie Droz

\section{Edizione cartacea}

Data di pubblicazione: 1 dicembre 2013

Paginazione: 111-154

ISBN: 978-2-600-01779-4

ISSN: 1423-3983

\section{Notizia bibliografica digitale}

Enrico Garavelli, « «L'erudita bottega di messer Claudio». Nuovi testi per il Reame della Virtù (Roma 1538) », Italique [Online], XVI | 2013, online dal 01 décembre 2016, consultato il 01 mai 2019. URL: http://journals.openedition.org/italique/372 ; DOI : 10.4000/italique.372 
E N R I C O GARA V E L L I

«L'ER U D I T A B O T T E G A

D I MESSER CLA U D O».

NUOVI TESTI PER IL

REAME DELLA VIRTì (ROMA I 5 3 8 ) 



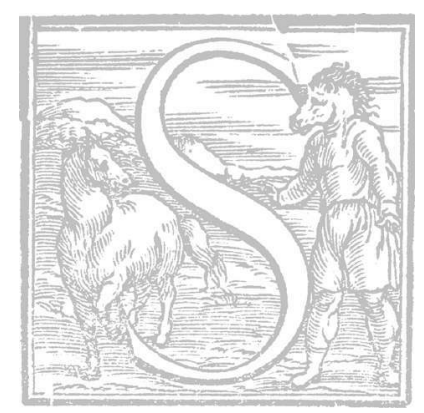

ulle modalità di aggregazione dei circoli letterari romani negli anni immediatamente successivi al Sacco, sulle loro funzioni, sui loro riti, sulla loro struttura fluida e aperta di cenacoli umanistici, molto è stato scritto. ${ }^{\mathrm{I}}$ Cosi come molto inchiostro è stato versato per delimitare nomi, luoghi, occasioni, programmi, irrigidendo la scarsa e per cosi dire carsica documentazione di cui disponiamo, con l'ambizione di ricostruire una disciplinata anagrafe accademica; operazione velleitaria, quando solo si consideri che appunto fino al I538 (o meglio, come vedremo, al I540) di accademia in senso stretto non si può nemmeno parlare. In questa sede, basterà ribadire che con il sodalizio di cui ci si occupa, la Virtù, si apre una "fase nuova della cultura romana»; ${ }^{2}$ una fase in cui si punta a riaffermare il primato di Roma recuperandone la tradizione classicista $e$ in cui si procede senza indugi sulla via di un nuovo, aggiornato umanesimo, lungo un vettore che infine trionferà nell'età che tradizionalmente si ama definire controriformistica. Esito forse paradossale, comunque per allora inatteso, se il successo della ratio studiorum comporterà anche la progressiva contrazione degli spazi riservati al comico e al riso. ${ }^{3}$

Ma se la Virtù costituisce davvero, come pare indubitabile, uno snodo fondamentale nella cultura romana di quegli anni, occorrerà evitare il rischio di applicare a quell'esperienza schemi interpretativi validi, forse, per gli anni immediatamente precedenti, ma per quella fase inadeguati. Inadeguato appare anzitutto l'ormai logoro paradigma critico adibito da quanti proiettano anacronisticamente su quelle sodalitates $i$ tratti della crisi del letterato di fine Ottocento o perfino dell'alienata condizione borghese, scissa tra l'angoscia di un quotidiano grigio e impiegatizio e la prepotente dimensione creativa e appagante della scrittura; un paradigma che non riconosce altra funzione alla letteratura se non quella di denuncia e di protesta; che divide rigidamente $i$ letterati in intellettuali organici a sistemi ingiusti e prevaricatori e scrittori indisciplinati e engagés, severi interpreti di una letteratura intesa come forma di apostolato civile. Tale indirizzo critico, che non a caso ba prodotto $i$ suoi frutti migliori tra la fine degli anni Sessanta e $i$ primi anni Settanta, ${ }^{4}$ appare oggi incapace di cogliere lo specificum della 
letteratura al di fuori della sua capacità di rappresentare o meno il mondo. Ravvisando nell'impegno tutto letterario dei nostri accademici (di una letterarietà indocile e polimorfa, ma che cede volentieri a Pasquino il compito di commentare l'attualità politica) null'altro che letteratura d'evasione, compensatoria, prodotta da scrittori nevrotici e repressi «per fuggir la mattana»,' questo schema interpretativo non può che approdare a un superficiale giudizio di gratuità e oziosità e a sbrigative denunce per atti osceni, confondendo la cifra retorica dei testi (la disposizione burlesca, il ricorso all'elogio paradossale, il versatile strumento della parodia, l'esibizione del corpo e del sesso, la tendenza alla deminutio, se non proprio un atteggiamento di aristocratico understatement) e $i$ loro obiettivi culturali, tutt'altro che disimpegnati e autoreferenziali; quasi si trattasse del chiacchiericcio serale di annoiati bancari o ragionieri. Sicché piace riscontrare una cauta, ma inequivocabile palinodia nelle considerazioni di un'allieva dei divulgatori di quel verbo, che in limine a un suo rilevante studio sull'elogio paradossale ha recentemente sottolineato "l'impegno serio ed etico dissimulato nello stravolgimento comico» e la «bizzarria mai fine a se stessa ma quasi sempre allusiva e antidogmatica» di quei contesti (al Caro si arriva facilmente attraverso la troppo spesso fraintesa lettera a Marcantonio Piccolomini in biasimo della scrittura, da rileggere tenendo sullo sfondo perlomeno il Fedro di Platone, Erasmo, Agrippa di Nettesheim, Ortensio Lando, il cosiddetto Opsimathes). ${ }^{6}$

In secondo luogo - ciò che più conta, in tempi in cui per fortuna la critica ideologica sembra aver perso terreno - credo che si debba fare attenzione a non applicare indebitamente ai nostri Virtuosi un altro teorema, sviluppato in particolare da Danilo Romei e Antonio Corsaro, ${ }^{7}$ che tende a leggere nella produzione di Berni e dei cosiddetti berneschi, «piu che $[u n]$ gioco distraente e consolatorio», una "poesia della difficile o impossibile evasione»; letteratura «negativa, dunque critica», capace di "roffrire momenti di aspra consapevolezza» e di "esprimere il malessere e le incertezze piuttosto che le vacanze e gli svaghi di una società letteraria». ${ }^{8}$ Se tale lettura mi sembra del tutto convincente nel caso appunto di Berni e della sua cerchia negli anni immediatamente successivi al Sacco (non a caso l'eccellente saggio di Romei si arresta al I537), mi pare per contro difficile applicare questa interpretazione alla brillante e spavalda stagione inaugurata dalla Virtù, in cui lo stanco ripiegamento degli epigoni (Molza, Bini) ${ }^{9}$ 
convive con la voce ambiziosa e sprezzante di una nuova generazione di letterati, e ne appare come sovrastata. Non vedo affatto tracce di malinconiche inquietudini o dei fantasmi persecutori di un passato godereccio e rimpianto nella lettera che Claudio Tolomei rivolge a Giulio Landi il 6 maggio I540 (per non dire dei suoi numerosi interventi parenetici ai Virtuosi): ${ }^{\text {10 }}$

mi si svegliò una certa speranza di creder che voi poteste venire insin a Roma, per riveder un poco gli amici vecchii, e per considerar, se questa città ha presa nuova forma di poi che la lasciaste. E se non altro per riguardar di nuovo queste antiche reliquie de le maraviglie Romane, e per goder questa aria, questo spirito, questo cielo, il qual da ogni parte par che spiri disiderio di virtù, e di gloria. ${ }^{11}$

E che dire di un sonetto di Giacomo Cenci ${ }^{-12}$ ad Alessandro Corvino, ${ }^{\mathrm{I}}$ in cui all'inchiesta «Di questo secol che vi pare?» si risponde enfaticamente «Da porlo io tra i più bei nol stimo indegno, | che fur già mai nel gemino hemispero»? ${ }^{14}$

Semmai, sarà vent'anni più tardi che si guarderà con malinconia a quella stagione piena di promesse, come certificano le parole di Dionigi Atanagi ${ }^{\text {is }}$ a commento di un suo componimento:

I quindici anni del Pontificato di Papa Paolo Terzo si posson dire tanti anni di secol d'oro: conciosiacosa che tutto quel tempo Roma godesse una pace veramente d'oro, piena di tranquillità, et senza alcuno turbamento, od affanno. Le virtù, le lettere, et tutte le arti liberali fiorirono [...] Levaronsi adunque in quel felicissimo tempo ne la città di Roma molte Academie di diversi elettissimi, et famosi ingegni, sì come furono quelle de la Virtù, de la Poesia nuova, de lo Studio de l'Architettura, de l'Amicitia, del Liceo, l'Amasea, et più altre. ${ }^{16}$

Insomma: Berni era un sopravvissuto dei papati medicei, Caro e Tolomei sono le nuove voci della Roma farnesiana.

Di fatto, le tematiche affrontate dai Virtuosi - certo, per lo più con attitudine parodizzante, ma la parodia è modalità complessa, capace tanto di demistificare quanto di ricanonizzare - abbracciano quasi l'intero ventaglio del dibattito culturale di quegli anni. Già Dionisotti aveva colto nel gruppo tosco-romano di Tolomei la «crescente insoddi- 
sfazione e impazienza dei limiti stretti in cui la riforma linguistica e letteraria operata dal Bembo aveva ridotto la poesia» e la «precisa e decisa volontà di rompere quei limiti sul versante della tradizione classica». ${ }^{17}$ In una serie di interventi sul Commento di Ser Agresto bo tentato di mostrare la centralità di quel testo nel progetto della sodalitas riunita appunto intorno a Tolomei. ${ }^{18}$ Un progetto che prende le mosse da un rifiuto radicale del canone dell'imitazione unica (si opta al contrario per un eclettismo che "apre" alla modernità, annettendosi il miglior Quattrocento toscano e favorendo gli apporti dei buoni scrittori viventi); che si mostra insofferente di fronte al paradigma platonizzante e non accetta di ridursi a diafana e rarefatta lirica erotica; che in fatto di lingua rifiuta tanto la duplice restrizione geografica e diacronica del fiorentinismo arcaizzante del Bembo quanto l'astratta indeterminatezza della «lingua comune»; che prende le distanze da quel «dolce zugo» del Berni ${ }^{\text {I9 }}$ e dai suoi prosecutori e viceversa esalta il petrarchismo non canonico del Molza, nutrito di succhi classici; che ribadisce il carattere colto, aristocratico e sostanzialmente elitario della pratica letteraria, contro la prepotente ascesa di mastri di stalla e "poetane»;; $;^{20}$ che parodizza la fluviale e recentissima pratica del commento ai nuovi classici, segnatamente ai Rerum vulgarium fragmenta, quale orpello e diaframma, indizio di una trivializzazione del rapporto con gli auctores della poesia volgare e latore surrettizio, in alcuni interpreti, di un vincolo indebito tra produzione letteraria ed esperienza biografica.

Anche gli altri testi superstiti, oltre a quelli che si producono per la prima volta in questa sede, dimostrano che $i$ contubernales della Virtù erano tutt'altro che animucce rintanate nelle loro camerelle, dove celebravano gli squallidi e compensatori riti di esistenze complessate e represse. ${ }^{21}$ La Cotognata del Bini, la Nasea e soprattutto la Diceria di S. Nafissa del Caro ci riportano agli entusiasmi di quegli anni per le «antiche reliquie de le maraviglie Romane» (il I538 è l'anno della traslazione della statua di Marco Aurelio sul Campidoglio) e sul nascente, rilevantissimo fenomeno del collezionismo. E in quel torno di anni che nascono le grandi collezioni farnesiane, ma anche le non meno significative raccolte di Giovanni Gaddi o Rodolfo Pio, per citare due prelati molto prossimi al sodalizio. ${ }^{22}$ Lo stesso Ippolito de' Medici, al cui servizio si erano incontrati per la prima volta Molza, Tolomei, Landi, Panciatichi e molti altri Virtuosi, si era dilettato di arte 
antica, sebbene più con l'acerba curiosità del collezionista dilettante che la matura consapevolezza dell'antiquario. ${ }^{23}$

Nella Cotognata, l'interruzione improvvisa del ternario d'avvio (dopo soli $13 \mathrm{vv}$ ) diventa occasione per una tirata contro $i$ «salta in panca, che vanno attorno», autori di versi che «fanno o rider, o pianger altrui»; e per una rivendicazione dell'importanza della prosa, «favellare sciolto» (oratio soluta) che ancora attende un'adeguata regolamentazione (dichiarazione di assoluto rilievo, se riflettiamo sugli imbarazzi del Bembo). Bini documenta inoltre la simultaneità delle cene della Virtù con gli esperimenti “barbari” del Tolomei («que' nuovi versi» che «havendo voce di prosa, et signification di versi, hanno cosi del retorico, come del poetico») e degli interessi filologici del gruppo su Vitruvio («il $T$ par ancor esso una colonna, che senza basa, et capitello sostenga un Architrave in billico, cosa non so se pensata mai, non che scritta da Vittruvio»). In quelle poche pagine c'è insomma in nuce il programma della cosiddetta Accademia della nuova poesia toscana e del Liceo di una celebre lettera di Luca Contile. ${ }^{24}$ Sempre la Cotognata costituisce una precoce testimonianza della crescente pratica delle imprese (Tolomei aveva chiesto ai "vassalli" di proporne una per la Virtù). ${ }^{25}$ Bini, infine, ancora per ottemperare a una prescrizione del re di turno, commenta un «terzetto» del Triumphus Cupidinis (III I57-59), ironizzando sulla pretesa riconducibilità della poesia di Petrarca alle sue fonti occitaniche (un libro «molto antico in lingua Spagnuola, tradotto di lingua Arabica d'un libro, che fu già portato del Regno di Feccia in Portogallo, et di Portogallo in non so che libreria di Spagna, gran tempo inanzi che fussi il Petrarca, et che venutane copia in Provenza a quelli Poeti, quivi allhora fu tradotto in lingua Toscana dal Petrarca, il quale con tutto che lo riducessi in quella dolcezza, che ognun sente, la invention però fu d'altri, et in lingua del primo autore stimano che fussi dolcissima»); ${ }^{26}$ passo in cui si intravedono in filigrana le pagine sulla poesia provenzale. $^{27}$ Lo stesso attacco al diluvio dei commenti petrarcheschi lo ritroviamo in una brevissima diceria di Giuseppe Cincio, che prende di mira il verso Et qual è 'l mel temprato con l'assentio [Tc III I87, da confrontare anche con Rvf CCXV I4], ironizzando sulla tecnica, davvero petrarchista, dei «contrapposti»; ${ }^{28}$ e in altre dicerie minori, meno interessanti, di Pier Paolo Gualtieri e Coriolano Martirano. ${ }^{29}$ 
Tutto ciò nel breve volgere di una stagione, diciamo pure il primo semestre del I538. Ripercorriamo dunque ancora una volta le tappe di quell'esperienza, già variamente declinate altrove. Il Io gennaio il Caro annuncia al Varchi un convivio festivo nel palazzo Gaddi di via Giulia per l'Epifania:

Questa sera [il Molza] sarà qui a cena, ché faremo il reame della befania, dove saranno di molti passatempi. ${ }^{30}$

È quasi certamente l'inizio della serie di incontri della Virtù, segnato, si può supporre, dall'incoronazione del primo re, l'anfitrione Giovanni Gaddi. ${ }^{31}$ Per quella serata fu probabilmente concepita la Diceria di S. Nafissa, offerta appunto al Gaddi, e verosimilmente alcuni testi tra quelli che si pubblicano qui di seguito ( $i$ più probabili sono $i$ nn. 3 e II). ${ }^{32}$ Il dopocena doveva essere accompagnato dalla diffusissima pratica del gioco d'azzardo. L'Aretino ci rivela che il Tolomei inventò un gioco «di sessanta carte» intitolato proprio Virtù (difficile dire di cosa si trattasse), che doveva allietare (o rattristare, assecondando $i$ moti dell'alterna fortuna) le serate dei contubernales. ${ }^{33}$ Nelle domeniche successive si avvicendarono diversi re e, per usare ancora le parole del Caro, «il Giuoco de la Virtù crebbe tanto che diventò reame». ${ }^{34}$ Possiamo stabilire, sulla base degli indizi sparsi nelle opere superstiti, questa cronologia: il secondo re fu il medico fiammingo Giuseppe Cincio (citato come tale nella Cotognata del Bini, che gli indirizzò l'omaggio di un bicchiere, accompagnato dal capitolo omonimo; il Tolomei gli rivolse un componimento al momento irreperto, il Dado), il terzo Claudio Tolomei (a lui sono indirizzati, oltre alla Cotognata, le

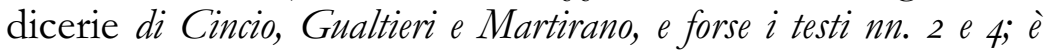
anche possibile che l'occasione abbia fornito al Caro e al Molza il primo spunto per il futuro Commento di Ser Agresto). ${ }^{35}$ Il quarto re, se accettiamo una problematica indicazione di Mattio Franzesi nel Capitolo del viaggio a Benedetto Busini, sarebbe stato Sebastiano del Piombo ("che mi sovvenne allor di fra Bastiano, / che questa sera assiso in maestà, / onorerà la fava a piena mano»). ${ }^{36}$ Il testo $n$. I che qui si pubblica permette di supporre che il quinto re sia stato il pistoiese Paolo Panciatichi (figura su cui occorrerà tornare). Mentre il sesto re fu Giovan Francesco Leoni, ${ }^{37}$ destinatario della Nasea del Caro (scritta prima del 10 marzo $)^{38}$ e della successiva Lettera al re Nasone, che 
reca la data I0 aprile I538. In quella stessa lettera, Caro rivela al Leoni che «il Regno de la Virtù è in declinazione», anzi - così a Bernardino Maffei in altra missiva dello stesso giorno - che addirittura «è sbandato». ${ }^{39}$ Sembrerebbe la fine di quell'esperienza: il gruppo si era infatti momentaneamente disperso, molti influenti cortigiani avendo seguito la corte papale a Bologna in vista dei colloqui di Nizza con Francesco I e Carlo V. Sennonché i testi nn. I e Io ci rivelano che tra la Nasea e la lettera al Leoni del Io aprile ci fu almeno un altro re, probabilmente l'antiquario Alessandro Corvino, ${ }^{4 \circ}$ o forse addirittura due (il secondo potrebbe essere Francesco Maria Molza). ${ }^{4 \mathrm{I}}$ Ma fu l'ultimo colpo di coda. L'anno successivo il Tolomei tornò a riunire saltuariamente $i$ compagni (il Caro, che fu ripetutamente assente da Roma, soggiornando prima a Napoli, poi in Romagna al servizio di Giovanni Guidiccioni, iniziò a defilarsi), concentrandosi però sul programma, già in fieri da tempo, della Nuova poesia. Il progetto, che spunta in molte lettere del Tolomei di quei mesi, si concretizzò, come è noto, nella pubblicazione dei Versi, et Regole de la nuova poesia toscana (Roma, Blado, ottobre I539). Solo nella primavera del I540 messer Claudio riusci a riorganizzare stabilmente la compagnia, regolamentandone appartenenza, gerarchia e rituali. É probabile che in questa fase il Tolomei abbia potuto sfruttare l'appoggio di Francesco Colonna, allora vescovo di Rossano, ${ }^{42}$ che è infatti celebrato in due componimenti del senese pubblicati venticinque anni più tardi dall'Atanagi. ${ }^{43}$ Il Regno della Virtù, contubernio itinerante, diventa allora un'accademia vera e propria, con tanto di sede stabile nella dimora del Colonna (probabilmente proprio nel palazzo di piazza SS. Apostoli). A questa seconda fase alludono due importanti documenti: il primo è una celebre lettera ancora del Caro del 20 maggio I540: il mittente dichiara al Leoni di non aver ricevuto la missiva con la quale l'amico gli annunciava la rinascita del sodalizio, ${ }^{44}$ e si scusa dunque per non aver trasmesso il suo omaggio al

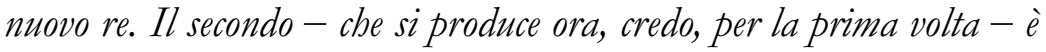
un libretto di 24 cc. pubblicato nel gennaio del I547 a Parma dallo stampatore Seth Viotto (molto vicino ai Farnese), e intitolato Due orazioni in lingua toscana. ${ }^{45}$ Camuffate sotto quel titolo generico compaiono due orazioni, contro e a favore di Giovan Francesco Leoni, segretario dell'Accademia; arringhe anonime ma senza dubbio da assegnare alla penna del Tolomei. ${ }^{46}$ Le due orazioni, in stile giudiziale, verosimilmente fittizie, riprendono naturalmente la tradizione sofistica 


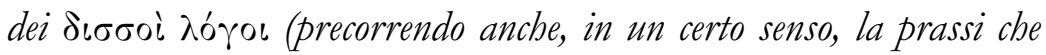
imperverserà nei collegi gesuitici): ${ }^{47}$ è il trionfo della retorica, che accompagna il processo di dilatazione degli spazi della $\delta o ́ \zeta \alpha$ ai danni della logica che aurà tanta fortuna a fine secolo. La materia del contendere è la divulgazione indebita di scritture accademiche da parte del Leoni, contro «i molti lodevoli ordini di questa virtuosa compagnia». Già "alzato al sommo principato» (cioè Re), scelto quale "Cancelliero», "anzi, Secretario, e perpetuo», dell'Accademia e depositario delle scritture, appunto, "segrete" ("le leggi, i decreti, gli ordini, l'bistorie, gli annali, e' santissimi misterij», in una parola, le «secrete aretologie»), il Leoni, secondo l'accusa, aveva promesso di trasmettere a certo «Fabritio» alcune composizioni, contro la legge dell'Accademia «che non vuole che sia lecito il publicar fuor della compagnia cosa alcuna». Al di là degli intenti ludici, ci troviamo ora, apparentemente, di fronte a un'accademia regolare e strutturata, ben diversa dall'improvvisato «Regno della Virtù» salutato con entusiasmo dal Caro due anni prima. Nell'orazione difensiva il Tolomei stesso si premura di segnare la distanza dall'esperienza del carnevale 1538 , indicato come «quel primo raccoglimento della virtù». Il Leoni avrebbe divulgato solo componimenti afferenti a quella prima stagione ("l'opere fatte [...] nel primo nascimento della virtù»), non ancora "tutelate" dalle leggi successive (la «legge nuova», cioè lo statuto dell'accademia). Per contro, la vertenza si colloca «nel principio del suo rinascimento», dunque, probabilmente, nella primavera del I540, se accettiamo l'indicazione della lettera del Caro al Leoni citata sopra. ${ }^{48}$ Più avanti, Tolomei distingue ancora tra $i$ «misterj» «che non si possono né debbono in tempo alcuno, né per alcuna occasione divolgar mai», cioè «i principij, il fondamento, gli ordini, le relationi, le imprese, le cerimonie, le leggi segrete, $i$ nomi ascosti, $i$ numeri sacri»; e $i$ «secondi misterj, che son posti ne' componimenti dell'epistole, de' discorsi, dell'orationi, dell'bistorie, delli epigrammi, delle ode, dell'elegie, et altre sorti di vaghe poesie». E suggella $i$ sottintesi esoterici del discorso con un colophon crittografico che mi è rimasto indecifrabile. ${ }^{49}$ Mette conto di ricordare che nel I547, quando licenziava le due orazioni, il Tolomei, già lettore di diritto civile tanti anni prima a Siena, era Presidente del Consiglio Superiore della Giustizia nel ducato farnesiano. 
Tra $i$ testi indebitamente rubricati tra $i$ prodotti dei Virtuosi figurano due notevoli dicerie: la Formaggiata di Giulio Landi ${ }^{\circ}$ e la Furfanteria di Jacopo Bonfadio. ${ }^{\text {I }}$

Per quanto riguarda la Formaggiata, è certo che fu stampata a Venezia da Gabriel Giolito de' Ferrari, senza indicazioni tipografiche ma con il colophon «STAMPata I IN Piasenza Per | Ser GrasSINO FORMAG $=\mid$ GIARO. $\mid$ M.D.XLII.». ${ }^{2}$ Tale data è stata revocata in dubbio da Massimo Baucia, che ha visto nell'iniziativa la mano di Anton Francesco Doni, e ha proposto di abbassarla «anche di poco», pur di salvaguardare il ruolo dello scrittore fiorentino, che arriva a Piacenza nell'inverno I542-43. Vale anche la pena di citare una lettera del Tolomei dell'II aprile I545 (ma le datazioni dell'epistolario di messer (laudio sono spesso insidiose), nella quale si accusa ricevuta della promessa dell'invio di un "formaggio», che altro non può essere, mi pare, che la Formaggiata stessa, sulla quale il destinatario sospende il giudizio fino a che non lo avrà gustato, cioè letto. ${ }^{33}$ Ad ogni buon conto, si tratta con ogni evidenza di un testo scritto non a Roma, ma a Piacenza («nondimeno è delle cose, che noi habbiamo qui megliori, et più care», c. 3v), lontano dall'Urbe ("io absente, et da lei [cioè dal Re della Virtì] cosi lontano», c. 3r) e con una cognizione tutto sommato approssimativa della Virtù, alla quale in un certo senso il Landi offre la propria adesione ideale («vorrei pur al vostro reame [...] approssimarmi», c. 3v, «supplicando la Maestà vostra, si degni, non dirò accettarmi per suo domestico et familiare, [...] ma solamente havermi, et connumerarmi per un servidore de gli altri valorosi, honorati, et fedeli servitori suoi), c. $I 7 v$ [recte $2 I \nu])$.

Tra gli elementi interni sparsi nel testo e fruibili per una datazione spicca una triplice menzione della Nasea del Caro (che, come si è visto, fu scritta nel carnevale del I538). ${ }^{54}$ A quanto pare, però, nel testo non si fa mai riferimento al Commento di Ser Agresto, cio che ha indotto Baucia a desumerne un termine ante quem (agosto I539, data della princeps). Se si incrociano questi dati con l'attestazione di Sere Stentato (nom de plûme dell'autore), che ricorda le «efficacissime parole» con le quali «mastro Ferrante» caldeggiò l'invio dell'operetta al destinatario ("quando la corte di sua beatitudine era in Piacenza», dunque tra I6 aprile e 3 maggio I538, c. I7v [recte 2Iv]), si puo ipotizzare che la Formaggiata sia stata redatta tra la primavera del I 38 e l'estate dell'anno seguente (con eventuali ritocchi 
successivi); dunque al di fuori dell'attività specifica del Reame della Virtù in senso stretto, e piuttosto per suggestione delle notizie che provenivano da Roma. Antico familiare di Ippolito de' Medici, come altri protagonisti della Virtù, il Landi sarà stato invitato a partecipare al gioco come una sorta di "socio corrispondente" (non a caso, il cardinale è menzionato come "padre dei Virtuosi» nella lettera introduttiva di Grassino Formaggiaro). Una lettera del Tolomei al Landi stesso da Roma, 6 giugno I543 conferma del resto la lunga assenza del gentiluomo piacentino dalla città dei Papi, iniziata verosimilmente con la scomparsa di Ippolito (morto, come è noto, il 10 agosto I535). ${ }^{55}$ Del cerimoniale accademico la Formaggiata condivide comunque giudiziosamente l'associazione dono più omaggio letterario («ognuno l'avea a presentar d'una stravaganza e d'una composizione»). ${ }^{56}$

Quanto alla «Sacra Corona» cui è destinata l'operetta, sembra persuasiva ancora un'ipotesi di Baucia, che vi ravisa Giovanni Gaddi. ${ }^{57}$ Tale proposta poggia soprattutto sullidentificazione di "mastro Ferrante» con il «messer Ferrante» di una lettera del Caro, ${ }^{58}$ certamente lo stesso personaggio che introduce Annibale nel gabinetto di antichità del palazzo di via Giulia descritto grottescamente nella Diceria di S. Nafissa, ${ }^{59}$ e cui probabilmente va assegnato il testo $n$. II che si pubblica qui sotto. Il Gaddi mori improvvisamente il is ottobre del I542, fatto che non può non essere messo in relazione con la data che figura sul frontespizio della giolitina e, in generale, con l'iniziativa della pubblicazione: sia che in questa coincidenza si debba ravvisare un'iniziativa celebrativa e nuncupatoria vanificata dalla scomparsa del destinatario, sia che, al contrario, vi si debba leggere un tardivo omaggio, in mortem, all'eminente prelato. ${ }^{60}$

La lettera «Il stampatore a lettori» (da attribuirsi dunque a «Ser Grassino Formaggiaro» anziché a «Sere Stentato», in un gioco di antitesi tra "stipsi" letteraria e abbondanza e naturalezza di cui era maestro l'Aretino) ${ }^{61}$ denuncia del resto immediatamente una divaricazione temporale tra redazione della Formaggiata e sua pubblicazione, ispirandosi apertamente alla lettera del Barbagrigia stampatore che apre la princeps del Commento di Ser Agresto (agosto I539). Tale lettera è contesa tra lo stesso Giulio Landi (Rhodes) e Doni (Baucia); ma quest'ultima proposta sembra scontrarsi con evidenze di tipo linguistico e stilistico di segno negativo, e presenta anche qualche 
difficoltà ad accordarsi con successive menzioni della Formaggiata in altre opere doniane. ${ }^{62}$

La Furfanteria, edita per la prima volta tra le Lettere facete dell'Atanagi senza attribuzione ("Di incerto autore») e con interventi censori fu restituita a Jacopo Bonfadio nel 1978 da Aulo Greco, che la ripubblicò sul manoscritto $A$ I3 inf. della biblioteca Ambrosiana di Milano. ${ }^{63}$ Il Greco ritenne di poterla connettere all'attività dei Virtuosi e la datò pertanto al carnevale del I538. Tuttavia, dei personaggi citati, solo Molza rimanda specificamente a quell'ambiente. Giomo ( (Giommo») parrebbe il pollaiolo fiorentino del capitolo del Lasca sulla tornatella, dei Marmi doniani e dell'XI salterello del Bronzino. ${ }^{64}$ Altri personaggi sono probabilmente invenzioni dell'autore: Matteo di Biello, Fuligni, Pateti, Bratti, Olgiato, la prostituta fiorentina Pullica e quella romana Culaccio, Martina, Panuzio, Siviana. Molte proposte di identificazione avanzate dall'ultimo editore sono poi per lo meno avventurose: se «il nostro furfante Biagio» può indubbiamente far pensare a Biagio Martinelli, il cerimoniere di Cesena ricordato da Berni e Caro, ormai quasi un personaggio letterario, che Titta Rosso possa essere identificato nel Rosso buffone di Ippolito de' Medici è ipotesi senza riscontri. Che poi "Lucazzo» sia Luca Martini (che non era nemmeno un Virtuoso!), "Mathia» Matteo Franzesi e addirittura lo "zio Modestino» il Caro (perché entrambi marchigiani) sembrano davvero pure illazioni. Certo, la scrittura, se non redatta, è almeno ambientata a Roma ("Romulo, fondator di questa Roma», "la nostra Corte Romana», rispettivamente pp. I63 e I66), ma cio che proprio non convince è la sua totale estraneità all' ambito semantico della Virtù e alla consuetudine accademica del dono di omaggio al re di turno. L'impressione è che la diceria altro non sia che un esercizio virtuosistico di variazioni e digressioni condotto sulla falsariga della Nasea, ma in forma del tutto irrelata rispetto ai rituali intercenali del sodalizio. Fa riflettere soprattutto un accenno contenuto in una lettera a Fortunato Martinengo da Padova, 24 novembre I543, nel quale il Bonfadio riferisce all'amico di aver avuto tra le mani una copia della Diceria di Santa Nafissa. "Sembra strano che un Virtuoso avesse avuto notizia della diceria solo nel I543. D'altra parte, nel seguito della lettera il Bonfadio rivela all' amico il desiderio di mettere in piedi «un'Accademia sulle rive del Benaco, o in Salo, o in Maderno, ovvero in Tusculano», probabilmente ispirandosi ai sodalizi romani che aveva forse solo sfio- 
rato. ${ }^{66}$ Infine, l'unico accenno noto al testo (su cui si fonda, peraltro, l'attribuzione) è contenuto in una lettera ancora al Martinengo, senza data ma non anteriore al I544: "V.S. si ricordi della furfantaria mia; io non burlava e quella la prese in burla». ${ }^{67}$ E sintomatico che il Greco abbia riferito «quella» alla Corte Romana, antecedente che mai figura nella lettera; mentre probabilmente il Bonfadio altro non fa che lamentare il suo stato miserabile di "furfante" (cioè mendicante, postulante) al Martinengo ("quella», cioè «V.S.»). La diceria sarà stata dunque scritta nel biennio I543-I 544 .

I testi pubblicati in questa sede consentono di aggiungere alcuni nomi al sodalizio dei Virtuosi in quel suo primo «nascimento». La figura più interessante è senza dubbio Paolo Panciatichi, rimatore pistoiese quasi interamente inedito, ${ }^{68}$ noto soprattutto per aver copiato di sua mano in un celebre codice della Forteguerriana una cinquantina di testi di Antonio Cammelli. ${ }^{9}$ Nato a Pistoia nel 1499, secondo alcuni sarebbe entrato anzitutto al servizio di Ferrante Gonzaga, di Ippolito d'Este e di altri signori minori (notizie desunte sostanzialmente da una lettura disinvolta e frettolosa dei suoi testi e percio dubbie). Il suo soggiorno romano incominciò ad ogni buon conto subito dopo il Sacco, quando ottenne dal Comune la «condotta») per la città eterna (25 ottobre I528). Fu intimo del Molza e familiare di Ippolito de' Medici - lo si desume da due lettere dello stesso Molza a Gandolfo Porrino e al figlio Camillo -, ${ }^{70}$ per poi passare verosimilmente tra le clientele farnesiane dopo la morte del cardinale. Nei primi anni Quaranta dovette rientrare a Pistoia, dove ricopri incarichi prestigiosi per il Comune, diventando nel Is7I Gonfaloniere della città. Di questa seconda fase della sua vita sono significative testimonianze una relazione a Cosimo de' Medici da Roma, 25 luglio 1553 e una lettera a Michelangelo del I maggio I56I. ${ }^{71}$ Mori il 9 gennaio I577.

Altro personaggio interessante che troviamo tra $i$ Virtuosi è Scipione Orsini, figlio di Aldobrandino dei conti di Pitigliano e nipote di Nicola, che le fonti spesso designano con l'appellativo «di Nicosia» desunto dal suo palazzo romano, poi sede del Collegio Clementino. Familiare di Paolo III (ne era uno stipendiato regolare, come dimostrano i registri della tesoreria segreta pubblicati da Léon Dorez), accompagnò Pier Luigi Farnese a Fano durante il famigerato viaggio in cui si consumò l'oltraggio a Cosimo Gheri. ${ }^{72}$ Oltre a dedicarsi alla carriera militare, 
in conformità alle tradizioni del casato, si dilettò di poesia: un suo sonetto indirizzato a Giacomo Cenci (Cencio gentile, in cui chiaro si vede) fu antologizzato da Dionigi Atanagi nelle Rime. ${ }^{73}$ Anton Francesco Raineri, cui fu molto legato, gli rivolse due dei suoi Cento sonetti (nn. ХХИІІ е ХХИІІІ) ${ }^{74}$ e lo stesso Tolomei gli indirizzò alcuni distici. ${ }^{75} \mathrm{Fu}$ in cordiale corrispondenza col Bembo, al quale sottopose alcune sue rime, ${ }^{76}$ oltreché, naturalmente, col Tolomei. ${ }^{77}$ Nel ${ }^{1556}$, infine, uno Scipione Orsini figura come guardiano della Confraternita del SS. Crocifisso in S. Marcello; ma potrebbe anche trattarsi di un omonimo. $^{78}$

Quasi ignoto è il "Vantagio» cui viene attribuito il testo n. 3. Probabilmente va identificato con il «Vantaggi» familiare di Giovanni Gaddi che il Caro menziona in una lettera al Varchi da Roma, Io dicembre I534. ${ }^{79}$ Dal testo pubblicato qui sotto sembra doversi desumere che avesse partecipato all'impresa di Tunisi, forse al seguito di Alfonso d'Avalos, Ferrante Gonzaga o qualche principe minore italiano.

Una sorprendente, ma non tanto, nuova acquisizione è infine rappresentata da Marcello Cervini (il «M. Marcello» del testo n. o). Il futuro Marcello II, che nel '35 aveva ricevuto gli ordini sacri, era dal '34 segretario di Alessandro Farnese. In quegli anni era impegnato in una rapida ascesa in curia: segretario alle lettere latine e protonotario apostolico, quando al cardinale era stata assegnata la direzione della Segreteria pontificia (I gennaio I538) era diventato uno dei più potenti curiali farnesiani. ${ }^{80}$ Della confidenza che aveva con il Caro è spia una lettera di questi al Varchi del Io dicembre Is34 («messer Marcello, quale è qui ogni giorno con noi, e riesce un valente giovane»); ${ }^{8 \mathrm{I}}$ e della sua intimità col Molza dice una lettera del Varchi stesso, che scrivendo al poeta modenese tre anni più tardi lo pregava di portare $i$ suoi saluti (e le sue scuse) al «molto gentile e amicissimo messer Marcello Cervini». ${ }^{82}$

I testi presentati in questa sede consentono dunque di incrementare sostanzialmente il corpus dei componimenti afferenti alla Virtù. Se escludiamo $i$ nn. 8 e 9, che testimoniano un certo impegno e una vena niente affatto disprezzabile, si tratta per lo più di frammenti di scarso valore letterario, testi avventizi e occasionali, conservati più che altro per scrupolo documentario. Minuzie, però, che non vanno sottovalutate, perché ci consentono di contestualizzare meglio quelle riunioni che si deve supporre serali e insieme ci convincono una volta di più dell'atten- 
zione che negli ambienti curiali si prestava a ciò che avveniva all'intorno $e$ al di fuori di un mondo che a torto si vorrebbe chiuso e autoreferenziale. Rimbalzano in questi versi scazonti, accanto alle ambizioni personali e alle frustrazioni private, la storia politica e militare, $i$ miti imperiali e le velleità francesi, le smanie dei bibliomani e il gusto per l'arte antica, la curiosità per l'alchimia e la fiducia (?) nella medicina naturale. Non serve molto altro per restituirci il ritratto fresco e vivace di una cerchia di amici, molti dei quali destinati di li a poco a ricoprire ruoli di rilievo nell'Italia dei decenni centrali del secolo.

\section{NOTA AI TESTI}

I testi nn. I-7 e 9-I8 sono prelevati dal codice segnato $M M 693$ (già $\Sigma_{\text {I }}$ sopra 2 [1]) della Biblioteca Comunale "Angelo Mai" di Bergamo $(B G)$. Si tratta di una cospicua miscellanea di qualche centinaio di carte, stese da varie mani e in epoche diverse (XVI-XVIII sec.), numerate a lapis modernamente, suddivisa in varie sezioni tematiche. Nella quinta sezione (titolata Varie e d'occasione) è incluso un fascicoletto di 8 ff., di scrittura medio-cinquecentesca, che presenta una filigrana raffigurante un agnello pasquale inscritto in un cerchio simile a BRIQUET 47-5O (in particolare 50, che rimanda a Roma I535-I564). Il copista era senza dubbio un settentrionale, se non forse addirittura un non italiano, come rivela ad abundantiam l'attitudine allo scempiamento (cortecia, invito, stano, leca, arosti, fritelle, apparechio, ampolina, facia, saco...) e, per contro, ai raddoppiamenti ipercorretti (Appollo, reccarla, stranne, caccare, pillulletta, faccendo, ellette, annello...); meno significativa la mancata anafonesi di gionse; arcaizzante, a questa data, la rappresentazione con l'intermissione di $-\mathrm{h}$ - etimologica o paraetimologica dell'occlusiva seguita da vocale centrale o velare (per esempio pocho, bocha, pregho). Il quaderno fu donato alla Biblioteca "Mai" nel IgI2 da Alessandro Roncalli, e dell'omaggio diede conto Achille Locatelli Milesi, Di un'accademia romana del sec. XVI, "Bollettino della civica Biblioteca di Bergamo», VI (I9I2), pp. 34-38 (vi si pubblica il testo n. I7). Il testimone ha tutta l'aria di essere un manufatto avventizio, ad uso personale, confezionato a breve distanza dalla redazione dei testi e da qualcuno molto prossimo al sodalizio. I testi raccolti costituiscono indubbiamente un corpus coeso e convincente, che rende autorevoli le attribuzioni proposte, al di là della deplorevole precarietà testuale nella quale i componimenti ci sono pervenuti. 
Il testo $n .8$ è invece esemplato dal codice $D 313$ della Biblioteca Forteguerri di Pistoia (P). Si tratta di un manoscritto ben noto agli studiosi di Antonio Cammelli detto il Pistoia, di cui tramanda numerosi componimenti, ma conserva anche testi, tra gli altri, di Francesco Maria Molza e Annibal Caro (tra l'altro, la Diceria di S. Nafissa, ff. I8or-I83v). Appartenuto nel Settecento al patrizio pistoiese Domizio Tonti (da cui il nome di "Codice Tonti"), il codice è autografo di Paolo Panciatichi. Benché la tradizione in rima del Panciatichi non sia stata né studiata né edita se non per minimi prelievi, sembra che P veicoli una lezione ancora provvisoria del corpus poetico del Panciatichi, poi ritrascritto calligraficamente nel codice $B \quad 176$ della stessa Forteguerriana. Per il componimento $n .8, P$ conserva una redazione diversa del testo tràdito da $B G$ (se non ho visto male, esso manca però in $B I 76)$. E verosimile che $P$ veicoli una redazione riveduta e corretta dall'autore, mentre $B_{G}$, dato il suo carattere monotematico, costituirebbe una raccolta occasionale, più vicina all'esecuzione "storica" dei testi.

$\operatorname{Da} P(f .239 v)$ esemplo anche $i$ testi $n$. I9, due epigrammi forse riconducibili all'attività del sodalizio; e n. 20, che figura tra le altre composizioni come una sorta di appendice, un omaggio all'attività del Panciatichi, benché abbia poche possibilità di essere ascritto all'attività carnascialesca dei Virtuosi, e documenti piuttosto rituali analoghi.

Preferendo evitare una massiccia, avventurosa ritoscanizzazione della veste linguistica dei testi esemplati da $B G$, li trascrivo in forma semidiplomatica, regolarizzando parcamente le maiuscole. L'autografia di P pone ovviamente minori problemi. Mi limito dunque a sciogliere le abbreviazioni più corrive senza indicazione ( $p$. es. q.sta $>$ questa) $e$ aggiungo tra parentesi aguzze qualche indispensabile intervento di integrazione $(<>)$ o espunzione $(><)$. In nota, rendo conto inoltre di qualche proposta congetturale.

| Ir | Di Ms. Leone al Re Pau. [Panciatichi] quinto

Fonte: $\mathrm{BG}$, f. I $r$. Il v. 7 dell'ottava è vistosamente ipometro anzi lacunoso in rima, probabilmente più per guasto di tradizione che per imperizia del versificatore, mia la congettura. Il dono offerto è la coperta di un libro, ciò che apre uno spiraglio sulla pratica della bibliofilia allignante nel milien dei Virtuosi e ben indagata da A. H. Hobson, Apollo and Pegasus. An enquiry 
into the formation and dispersal of a Renaissance Library, Amsterdam, Gérard Th. Van Heusden, I975.

Caro signore, s'avertirete bene al mio pocho saper, direte el dono ch'io porto questa sera, si conviene solo a me darlo, e s'egli havrà del bono o non, si m'inganassi come aviene basta dimostri che servo vi sono $<$. $>$ D'un libro la cortecia <vi fo parte.> che altri vi daran le dotte carte.

2

Di Ms. Gian Fr. [Leoni] Anconitano

Fonte: $\mathrm{BG}, \mathrm{f}$. I $r$.

Del fonte d'Helicona almo et divino porgemi Appollo che 'l bel canto impari et tu signor del tuo purpureo vino ch'alzi 'l mio ingegno, et la mia voce schiari. Ch'io sento 'l Tebro dir longe et vicino non son tuttj i valor, tutt' $i^{83}$ Re pari. Dir di sì invito Re, sì eccelso Regno altra voce bisogna et altro ingegno.

| IV | Il Vantagio

Fonte: $\mathrm{B} G$, f. I $v$. L'autore, come s'è detto, è forse il Vantaggi che nel I 534 figurava tra i familiari di Giovanni Gaddi, che potrebbe essere il destinatario dell'omaggio.

Signor io vengo là da quelle partj ${ }^{84}$ ove stano i Pigmei piccoli et brutti onde insin qua pensai lieto portartj o d'arte o di natura opere et fruttj. Comprai un pappagallo per donarti et una gabbia ove li tengon tuttj. Pigli hor la gabbia vostra signoria che 'l pappagallo è morto per la via. 


\section{Di Ms. Scipione [Orsini]}

Fonte: $\mathrm{B} G, \mathrm{f}$. I $v$. Con ogni probabilità, il componimento esprime, come il n. 2, il tema dell'invito a riempire il calice per il brindisi: «questa» (v. 6) sarebbe dunque la coppa. Se questi due componimenti fanno sistema con il n. i 4 (o con il i 8) potrebbero essere indirizzati a Claudio Tolomei.

De la vostra virtù che 'l mondo accende surge o signor inestinguibil foco ${ }^{85}$ ove scaldar può l'anima che attende dal terren freddo alzarla in alto loco. La mia, che sempre oscura in basso scende cieca vi porge questa, et brama un poco del liquor, di cui basta una sol stilla: de l'invisibil lume una favilla.

| 2r| Di M. ${ }^{\text {ro }}$ Joseph [Cincio]

Fonte: $\mathrm{BG}$, f. $2 r-v$. Da notare l'incipit che parodia $R v f$ CXxIx e il riferimento finale, che sembra strizzare l'occhio alla Mandragola.

Di valle in valle, et d'uno in altro monte magnanimo s. ${ }^{\text {or }}$ io sono andato per cercar herbe et barbe che racconte sono da qualche author degno et pregiato. Queste in piazza rotonda o vero in ponte non trovarete, et in nessun altro lato $\mathrm{n}<$ é> ognun ${ }^{86}$ conosce il dauco ${ }^{87}$ o l'asphodelo né 'l vigor che li dà la terra e 'l cielo.

Di questa se ne fate un argomento ${ }^{88}$ ha virtù grande et è cosa Reale che vi caccia del corpo tutto 'l vento e gl'humor tristi che fariano il male. Un cristier val tant'oro et tant'argento et non si pò stimar, un mondo vale, massime quando il medico a ordinarlo è bon maestro, et lo spetial a farlo. 
Al capo, a gli occhi, al stomaco, alle reni son quest'altre utilissime et son bone $|2 \mathrm{~V}|$ massime si son carichi et son pieni d'humor, a cui bisogni purgagione. Questa ne' molli et ben colti terrenj si pianta et cresce, e non ha paragone per che distesa di dentro a i lenzoli è bona per le donne a far figlioli.

\section{Di M. Marcello [Cervini]}

Fonte: BG, f. I $v$. Il futuro Marcello II dona al re di turno nientemeno che... la pietra filosofale. Degli interessi del Cervini per l'alchimia sembra restare traccia nel Symb. Lx di Achille Bocchi, Concipiunt ignes specularia concava Solis, indirizzato appunto a Marcello (lo vedo in AchiLLE BосchI, Symbolicarum Quaestionum, de universo genere, quas serio ludebat, libri quinque, Bononiae, Apud Societatem Typographiae Bononiensis, I 574, pp. CXXVIII-CXXIX).

Questa ti dono o Re de gli altri Illustri più pretiosa assai d'ogni diamante che in molte parti per molt'anni e lustri cercat'ho per reccarla a te davante.

Ben che d'ogni vertù risplenda et lustri quest'una ti vò dir fra tante et tante che con essa potrai senz'altro augurio far di ramo ${ }^{89}$ oro et congelar Mercurio.

Di Ms. Pau. del Gover. ${ }^{\text {re }}$

Fonte: BG, f. $2 v-3$ r. Per quanto riguarda l'autore, si potrebbe pensare a Paolo Panciatichi, che per un certo periodo fu al servizio di Paolo Pallavicino governatore di Anagni (così sembra attestare, almeno, la Lettera del Palandrano a M. Orazio Marchiani pubblicata dal Fanfani: Paolo Panciatichi, «Il piovano Arlotto. Capricci mensuali di una brigata di begliumori», I, I 858 , pp. 2I7-2I). Il componimento manca però, se non ho visto male, nei due manoscritti autografi del Panciatichi alla Forteguerriana (D 3 I 3 
e B I 76). Inoltre, al v. 7 l'autore si dichiara esperto di medicamenti, sicché è probabile che si trattasse di un medico. Governatore di Roma era allora Filippo Archinto. ${ }^{90}$

\section{Veggio questi segnor Sacra Corona}

diverse et stranne cose presentare

chi per ornar vostra real persona

chi per darli piacer $<$, $>$ chi per mangiare. $\left|3^{r}\right|$

Può far il mondo che non si raggiona

di quel ch'importa più, cioè il caccare?

Però come dott'io di medicina

porto una pillulletta masticina. ${ }^{9 \mathrm{I}}$

Sopra un tegame di Pau. Panc. ${ }^{\text {co }}$

Fonte: P, ff. 35v-36r. Semplicemente «Del Panciatico» in BG. Queste le numerose varianti di BG, ff. $2 v$-3r, alcune delle quali verosimilmente testimoniano una redazione anteriore (ometto le varianti puramente grafiche): I delli; 4 com'io; 6 prompto; 8 di altrui; 9 obedir; I I dimostri] gli è 'l nome; I 2 faccendo; q(ue)sto; I 3 tuo nome] tua lode; I 4 sparsa, per ciò che colui che succese; 16 giugne; roba, forza, et oro; 18 splende in] è come; I9 rossetto un poco più, et è sì bono; 20 vivande; incita; 2 I Obbligato; 22 leca; 23 arosti; 24 honor del mondo et gloria del Reame; 26 opportuno; 27 spinace vi cuoce; 28 con fritelle; 29 son singular pel Re et cose ellette; 30 che chi volesse dirle; 3 I luongo sarebbe e'n cotal apparechio; 32 Mal no(n) vi staren le brache. Rispetto all'autografo, ho diviso mostril > mostr'il (v. 5) e regolarizzato la grafia di cuoce (due volte quoce, vv. 23 e 27 ).

Il tegame rappresenta per il Panciatichi un alias poetico, sfruttato anche in altri componimenti, a imitazione dell'Orto del Bini, rigorosamente peraltro eterosessuale (pentole e padelle alludono abitualmente al sesso femminile). L'oggetto in questione sarà stato un paiolo di rame («un po’ più rossetto», v. I9).

Inclito re delli altri Re splendore, a cui par che s'inchini ogni persona, che sol per dimostrar fede et Amore sì come debbo a tua Sacra Corona 
con gli altri vengo acciò mostr'il mio core

pronto in servirti, per che già risuona

il nome tuo famoso et singulare

d'altri superior, non dico pare.

Così venendo ad obbedir ti porto come è costume un pegno di mia fede che se come dimostri fia accorto facendo il successor di quello erede sarà tuo nome da l'occaso a l'orto sparso per tutto, perché chi succede loda quel Re, et tienlo almo et decoro ch'al Regno aggiugne forza, robba, et oro.

Ti porto dico questo vaso in dono la cui materia splende in calamita e un po' più rossetto, ma sì buono che a far vivande ciascheduno invita. So tu dirai: - Obligato ti sono quando adverrà te ne lecchi le dita delli arrosti che cuoce tal tegame et sarà l'ornamento del Reame.

Puossi operare a mille altre cosette secondo il tempo che fia oportuno. Chi spinaci ci cuoce, et chi lo mette in forno a far frittelle, ch'a digiuno si mangion che pel Re son cose elette et altre ch'a narrarle ad uno ad uno lungo sarìa. Ma tienlo che sia specchio mettendovi le brache del Re vecchio.

\section{Di M. Gandolfo [Porrino]}

Fonte: BG, f. $3 v-5 r$. Su Gandolfo Porrino, notevole rimatore modenese, mi limito a rimandare alla nota biografica apposta da Laura Sguazzabia alla sua edizione delle Stanze per Giulia Gonzaga, Parma, Università di Parma, 2000, pp. 47-6r; e a una comunicazione di D. Chiodo, Di alcune curiose chiose a un esemplare delle "Rime" di Gandolfo Porrino custodito nel fondo 
Cian, "Giornale Storico della Letteratura italiana», CXx, vol. CLXXx, fasc. 589 (2003), pp. 86-ıог. Se non è dubbio che l'oggetto donato fosse un anello, resta problematico il destinatario. Si tratta sicuramente di un modenese («gionse là dove voi nascesti et io», v. Io; lo stesso accenno alla chiesa di San Geminiano, patrono di Modena, v. 2 I, rimanda alla toponomastica della città emiliana), ciò che farebbe pensare al Molza. Sennonché l'auspicio finale a un'elevazione al rango cardinalizio desta qualche perplessità; sebbene in una stagione in cui perfino un Aretino poteva covare legittime aspirazioni alla porpora (sulla questione: P. Procaccioli, Un cappello per il divino. Note sul miraggio cardinalesco di Pietro Aretino, in Studi sul Rinascimento italiano / Italian Renaissance Studies. In memoria di Giovanni Aquilecchia, a c. di A. Romano e P. Procaccioli, Manziana, Vecchiarelli, 2005, pp. I 89-226). Considerando, inoltre, il riferimento al cappello verde (v. 48), che in araldica comporta uno stato ecclesiastico minore (abate, arciprete) di cui non abbiamo notizie riguardo al poeta (che godeva bensì di un cavalierato di S. Pietro), l'ipotesi pare ardua. La stessa immagine, in effetti, è utilizzata dall'Atanagi in una lettera a Tommaso Spica, Roma, I7 agosto I 449 («Voi sapete, quanto agevole sia a convertirsi quel capel verde in rosso, massimamente quando è in capo d'un huom così degno, come è Monsignor Tolomei», Delle lettere facete, et piacevoli [...] libro secondo cit., p. 95); ma come auspicio «di maggior grado» (dunque il cappello verde è quello vescovile che il Tolomei avrebbe ricevuto di lì a poco).

Un'alternativa non meno problematica chiama in causa Alessandro Corvino, che tutte le fonti dicono gentiluomo romano, salvo la Diceria di S. Nafissa del Caro, che lo accomuna a Molza e Porrino («Il Maroniano [= Molza], il Corvino e 'l Gandolfo, i tre chiarissimi Modanesi», in Annibal Caro, Gli straccioni commedia. La Ficheide comento. La Nasea e La statua della Foia [...], Milano, G. Daelli e C., I 863, p. I84). Se l'ipotesi dovesse reggere (ma nelle cronache modenesi non trovo traccia di famiglie Corvino, Corvini e simili), meglio si spiegherebbe la scelta del dono: il testo successivo (n. Io) documentando nello stemma familiare del Corvino - ma anche questa è un'ipotesi - la presenza di un corvo con un anello nel becco. L'affabulazione delle ottave sembra sfruttare ancora una volta un tema machiavelliano, l'escursione diabolica extra inferos della novella di Belfagor arcidivolo; che era però ancora inedita.

Saper dovete che l'antico Gigi poi che fu morto se n'andò a l'inferno.

Ma venendoli in odio i Regni stigi 
n'uscì per gratia di chi n'ha il governo. $|4 r|$

Et rittornato al mondo in panni bigi

longamente s'ascose et state et verno,

et portò sempre seco il cattivello

et vivo et morto il suo fatal anello.

Col qual andando in quella parte e in questa

gionse là dove voi nascesti et io

et rimirando una donzella honesta

di lei tutto s'accese nel desio,

et al fin se li cacciò sotto la vesta

non si pensando di pagar il fio <.>

Ma capitando quivi un gran Marchese $\mathrm{9}^{9^{2}}$

s'accorse che lo spirto era in paese.

Onde como fu noto il caso strano tosto si ragunò molta brigata et al fin venne un valente Capellano gridando forte: - Madonna è spiritata!

Presto menata sia in san Giminiano ch'io vò pigliar quest'anima dannata -. Così fu fatto, hor pensate signori di che voglia era Gigi a quel romore. $|4 v|$

Cominciando lo spirto a scongiurare subito fe' venire una ampolina et disse: - Ivi entro lo voglio cacciare -. Esser parea a Gigi in gelatina onde provando di poter scampare si trovò chiuso in quella caraffina dove de' fati altrui rese ragione al Capellan di cui restò prigione.

Onde se volse uscir de l'altrui porte per taglia bisognò pagar l'anello a quel bon prete, il qual venendo a morte e havendo me per compagno et fratello come volse fortuna et la mia sorte per testamento mi lasciò il gioiello et sempre l'ho tenuto per suo amore: hora lo dono a te, degno signore. 
Et pregho ben che la tua maestade miri il mio core et non sì picciol dono et prenda in grado che in mille contrade spargendo vo de le tue lode il suono. $|s r|$ Et come di virtude et di bontade tutti altri avanzi che nel mondo sono, così colui ch'<h>a per insegna il Giglio ${ }^{93}$ il tuo verde capel facia vermiglio.

IO

\section{L'Horto di M. Bini}

Fonte: $\mathrm{Bg}, \mathrm{f}$. $5 r$. Il destinatario è forse Alessandro Corvino. Per quanto non se ne conosca con certezza né l'estrazione sociale né l'eventuale stemma familiare, è verosimile che, fosse tradizione autentica o ricostruzione burlesca, nel suo blasone figurasse un corvo che stringe nel becco un anello; simbologia ben nota della famiglia magiara degli Hunyadi, il cui più noto rappresentante fu il re-bibliofilo Mattia Corvino.

Sire i $<$ '> ho inteso che voi havete un corbo nella vostr'arme, che tiene un annello in bocha, ove una corbezzola, un sorbo ${ }^{94}$ campeggerebbe meglio assai che quello sì come pò veder chi non è orbo. Però de l'un e l'altro, un ramicello vi mando, et ancho havrei mandato il frutto se il temporal non andassi sì sciutto.

Di M. ${ }^{\text {ro }}$ Ferante

Fonte: $\mathrm{B}$, f, 5 r. Si tratta probabilmente del Messer Ferrante al servizio di Giovanni Gaddi (vedi supra, p. I I 8), piuttosto che dell'archiatra pontificio Ferdinando Balami, pure lui Virtuoso. Non è necessario assumere che proprio il Gaddi sia il destinatario dell'omaggio, ma in assenza di altri elementi resta il candidato più verisimile.

Questo chiuso95 in or fin saldo diamante di che l'annel che v'ho portato è adorno 
mostra il cor vostro nobile et constante con le rare virtù ch'è ${ }^{96}$ cinto intorno. Et sì come alle gemme tutte quante con la finezza sua fa sempre scorno così al parangon di vostr'altezza signor ogn'altro è di minor grandezza. $|5 v|$

Del Padre [?]

Fonte: BG, f. $5 v$. L'autore dichiara di aver partecipato, con un ruolo di primo piano (v. 9), all'impresa di Tunisi del luglio i 535 ; se non si tratta di una millanteria, che sfrutta l'eco di quell'impresa e ne ridimensiona il mito. Non ho candidature da proporre, se non forse quelle di Scipione Orsini (uomo d'armi) e, in subordine, di Paolo Panciatichi, che per qualche tempo avrebbe servito Ferrante Gonzaga, presente all'impresa. Il testo non è però né in $\mathrm{P}$ né nel codice $\mathrm{B}$ i 76 della Forteguerriana (salvo errore). Il destinatario potrebbe essere il Leoni, che - come si è visto sopra - Tolomei ci presenta nelle vesti di segretario e conservatore dell'«opre» dei Virtuosi (v. I 5 ).

Nel tempo già che Carlo Imperadore andò con grande armata alla Goletta per discacciar quel ladro traditore di Barbarossa, ${ }^{97}$ et farne aspra vendetta nel furor di quel saco et nel rumore io mi trovai et presi una cassetta piena di gioie et di molte altre cose che son di stima al mondo et pretiose.

Le gioie comparti ${ }^{8}$ fra' capitani che si trovarno meco alla giornata et che menorno molto ben le $\operatorname{man}>_{\mathrm{n}}<\mathrm{j}$ contra quella canaglia disperata.

La cassetta signor fra' Cristian $>_{\mathrm{n}}<\mathrm{i}$ mai più vaga o più bella non è stata. Riponvi l'opre d'ogni tuo vassallo che s'assomigli a Glaudio, al Molz $>\mathrm{z}<\mathrm{a}$, al Gallo. ${ }^{99}$ 
Del Molz $>_{\mathrm{z}}<\mathrm{a}$

Fonte: BG, ff. $5 v-6 r$. L'autenticità dell'attribuzione è confermata dalla ripresa di alcuni moduli espressivi molziani attestati altrove: l'incipit ricalca il v. I 2 del sonetto Sacri intelletti, cui l'un tempio onora (esattamente: «Altri di gemme vi coroni, e d'oro»), la clausola del v. 7 riecheggia il v. 3 della prima delle Stanze sopra il ritratto di Giulia Gonzaga («Se così dato a i vostri tempi Omero | avesse il Ciel, come v’ha fatto bella | a suo diletto, e degna d'alto impero»). Cito da Francesco Maria Molza, Delle poesie volgari e latine, [a c. di P. Serassi], Bergamo, Appresso Pietro Lancellotti, I 747, vol. I, pp. I 3 e i 35 . Il dono offerto al re di turno doveva essere una falda, parte dell'armatura che proteggeva la zona inguinale al di sotto della panziera, o qualche altra forma di difesa delle parti basse (la «stanza della farda nel sampero» del v. 8 indica semplicemente i testicoli, deposito del liquido seminale); a meno che non si tratti proprio delle «brache del Re vecchio» menzionate dal Panciatichi (supra, n. 8, v. 32).

Altri di gemme vi coroni o d'oro invittissima sacra maestate ${ }^{100}$ o vi tessa di mirto o pur d'alloro con gloria eterna foglie alme et pregiate $|6 r|$ che vinto credo non serò da loro se all'eccellenza del mio don mirate real salvezza et degna d'alto impero ch'è $^{\text {IOI }}$ stanza de la farda nel sampero. ${ }^{\text {IOz }}$

Il vaso, di M. Claudio Tol[omei]

Fonte: $\mathrm{BG}_{\mathrm{G}}$ f. $6 r$. Mi sembra probabile che questo componimento si riferisca alla rinascita del sodalizio sotto il patrocinio di Francesco Colonna (che sarebbe il re e il destinatario del testo). L'avvio della seconda ottava è infatti molto vicino alla porzione centrale degli sciolti pubblicati dall'Atanagi (Spirti nobili, che felicemente, in De le rime di diversi nobili poeti toscani cit., vol. II, c. 3 I r ) : «Ecco c'hoggi risorge più di prima | la virtute honorata in ogni parte, | et qual bella Fenice già rinuova | più 
saldo il volo, l'indorate piume, | che posto ha 'l nido ne la gran colonna» (vv. 8-I 2).

Già di sacro liquor fui colmo et pieno che a più gentili spirti, fu più caro perché 'n lui per purgarsi dal tereno fango, con voler pronto si lavaro. Poi ch'amor di virtù qui venne meno chi s'attufasse in lui fu nullo, o raro chè 'l lusingar de l'aversario mio a ciascun di ben far tolse il desio.

Hora che sotto il vostro amato regno rissorge di virtù sì saldo amore, ove ogn'alma gentil par ch'abbia a sdegno i pensier vili, et che sol brami honore per esser da voi pien lieto a voi vegno de l'invisibil vostro almo liquore per che ogni spirto in quel lavato poi a sé virtù procacci, et gloria a voi. $|6 v|$

Di M. Cl[audio Tolomei] in nome de tuttj li vassallj

Fonte: $\mathrm{B}_{\mathrm{G}}$, f. $6 v$. Esempio unico di dono collettivo, l'omaggio in questione dovrebbe essere una sorta di sfera armillare. La Formaggiata del Landi ci rivela che il re di turno (cioè Giovanni Gaddi) aveva «studiato Euclide, la sfera, \& Tolomeo» (c. 7v); sicché il prelato fiorentino potrebbe essere il destinatario del dono.

Per che quella virtù che 'l vostro Regno

formò, del Cielo è simulacro in terra questo noi vi porgiam vero dissegno de' cerchi Eterni, ov'ogni Astrologo erra. Quivi è de la virtù con chiaro segno il colmo fuore, et meglio entro si serra per che vedrete aprendo questa sphera il vivo essempio de la virtù vera. 
Il Re allj vassallj

Fonti: Bg, ff. $6 v-7 r$, Francesco Maria Molza, Delle poesie volgari e latine, [a c. di P. Serassi], In Bergamo, appresso Pietro Lancellotti, I754, vol. III, pp. I47-I48 (= M). Queste le varianti: 2 augustiis M; 8 neve ullis M; 9

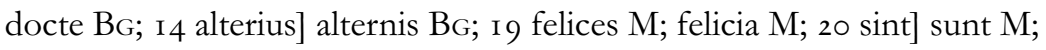
22 pendeat] fluctuat M. La copia utilizzata dal Serassi (che senza dubbio intervenne pesantemente sulla grafia) presentava una probabile banalizzazione al v. 20; lascio dunque in apparato anche l'adiafora del v. 22 (che potrebbe essere una variante d'autore ed è più vicina all'usus virgiliano). L'oscillazione alterius / alternis (v. I 4), data la prossimità paleografica delle due forme, deve invece necessariamente risolversi a favore o dell'una o dell'altra: opto per la lezione di $\mathrm{M}$, che mi sembra più adatta al contesto che esalta la reciprocità dell'amore tra i sodales.

Il fatto che questi distici elegiaci si trovino incassati in una sequenza di testi sicuramente di Claudio Tolomei (nn. I4, i 5, i 7 e i 8) e l'intonazione parenetica, accostabile a tanti scritti affini di messer Claudio (il v. ı。, d'altronde, allude chiaramente alla Nuova Poesia), consiglierebbe di assegnare anche questo componimento al senese; ipotesi cui non osta l'attribuzione al Molza nell'insidiosa raccolta settecentesca del Serassi (si veda il caso dei frammenti della traduzione del I Idillio di Teocrito del Caro in Francesco Maria Molza, Delle poesie volgari e latine cit., vol. III, pp. I4-I6; E. Garavelli, Il I Idillio di Teocrito tradotto da Annibal Caro, «Aevum», LXIX, I 995, pp. 555-9I). Non si capisce però per quale ragione il Tolomei, cui non furono mai congeniali esercizi consimili, sentisse l'esigenza di salutare i vassalli del regno in latino; ed è d'altra parte possibile, ma tutto sommato improbabile (non vedo connessioni tra $\mathrm{i}$ due testi), che $\mathrm{i}$ seguenti distici costituiscano un dittico con il precedente componimento; il che comporterebbe automaticamente l'esclusione di messer Claudio. Che il Molza sia stato Re della Virtù potrebbe suggerirlo, come si è visto, il componimento n. 9 del Porrino; ma l'ipotesi non è delle più probabili. Difficile, in mancanza di altri elementi, risolvere la questione. Non ebbe invece dubbi F. Baiocchi, Sulle poesie latine di Francesco M. Molza, Pisa, Nistri, I 904, pp. 83-84, che però lavorava fiducioso sull'edizione Serassi e non conosceva BG.

Magnanimi proceres, quorum haec virtute tenemus regna, ex augustis maxima principijs:

quo primum laudem vos nomine? quaeve rependam digna satis vestris carmina pro meritis? 
Vos regni decus, et columen, vos maxime nostro tantum hoc splendoris additis imperio.

Nam nullis aeque terra Itala gaudet alumnis nec ullis effert se magis ingenijs; sive graves tractet quis doctae Palladis artes seu texat stricto carmina vincta pede

et Regum magnas illustret carmine laudes vel ludat pugnas saeve Cupido tuas. $|7 r|$

Quid memorem ut dulcem inter nos spiramus amorem?

Et quisque alterius subiacet imperijs?

Et vos regna manent, regnique insigne corona, cuique suo cinget tempora vestra die. Atque ego qui nutu inter vos nunc cuncta guberno iam cupio vestro subdere colla iugo.

Foelices nimium Reges, foelicia Regna.

Talia ubi in ${ }^{103}$ Regem sint procerum studia atque is qui regno inter vos ${ }^{104}$ rerumque potitur summa, animi dubius pendeat usque ${ }^{\mathrm{IOS}}$ tamen. An vobis, tali ingenio, iucundius ille imperet, an vestro pareat imperio.

Epigramma del Regno de’ Virtuosi di M. Cl[audio]

Fonte: $\mathrm{B} G, \mathrm{f} .7^{r-\nu}$ (edito in Locatelli Milesi, Di un'Accademia romana del sec. $X V T$, cit., p. 38$)$.

Mentre i' miro in questi sacri spirti; e 'n queste figure parmi vederli Dei, parmi vederli homini.

Né so se gli homini de' dij alti han tolta la mente, o s'hanno a gli homini tolta la forma i Dej.

S’al Re nostro poi, Re grande, mia alma rivolgo non dubito, anzi dico, son Dij loro, esso Giove.

Sì di sacri ingegni ben adornasi questa corona ${ }^{\text {106 }}$ sì ben santamente governali ei $|7 v|$

ch'è Giove, chi ha tanta bontà, chi tanta potenza divino è spirito chi lo fruisce poi.

Dunque vive o Terren Giove sempre, et giovane sempre et tu sempre mai schiera beata vive. 
[Claudio Tolomei,] Presente di un pasticcio et un fiasco di vino

Fonte: $\mathrm{BG}, \mathrm{f} .7 v$.

\author{
Questi vasi ${ }^{107}$ vi manda il Tolomeo \\ di nettar l'un, l'altro d'ambrosia pieno, \\ né vuol come Platon volse, o Museo \\ che 'l contemplar del vero uno habbia in seno, \\ l'altro il piacer che nascere il ver feo \\ ché questo è poco vero, et quello è meno. \\ Ma dice, che con vero, et con piacere \\ l'uno è buono a mangiar, et l'altro a bere.
}

[Paolo Panciatichi, Due epigrammi]

Fonte: P, f. I $82 v$. I due epigrammi che seguono sono annotati subito dopo la copia della Diceria di S. Nafissa del Caro conservata nel codice Tonti. Autografi del Panciatichi (pasquinista ed epigrammista abituale), sembrano riferirsi proprio all'attività del sodalizio: il primo potrebbe aver accompagnato il dono di uno specchio, il secondo sembrerebbe una sorta di motto del sodalizio, giusta l'inchiesta promossa dal Tolomei. Dell'interesse per gli specchi nella nostra compagnia testimonia la curiosa vicenda di uno specchio ritrovato sull'Esquilino e passato per le mani di Livia Colonna e Alessandro Corvino e finito poi nelle collezioni farnesiane (R. Lanciani, Storia degli scavi di Roma e notizie intorno le collezioni romane di antichità, Roma, Quasars, I990, vol. II, p. 252). Così descrive la storia dell'oggetto Pirro Ligorio in una lettera a Fulvio Orsini del I4 febbraio i 563 : «fo fede a V. ${ }^{\text {a }}$. ${ }^{\text {ia }}$ del specchio che fu della bona memoria del Corvino, è antico, trovato dentro certi muri con ornamento di legno di larice molto grave, fu prima trovato da Giovanbellino pittore nella sua Vigna nell'Esquilie; nella morte del pittore venne alle mani del S. ${ }^{\text {or }}$ Martio Colonna, poscia dopo la morte della S. ${ }^{\text {ra }}$ Livia sua mogliere il Corvino procurò di haverlo con $>$ con $<$ quella sua solita et naturale ansietà e diligentia, havendolo acquistato, gli tolse via il suo ornamento il fece purificare nel lustro al specchiaro che è oltre per la via di san Salvator del Lauro, et l'ornò di quel'ornamento che hora tiene attorno» (Città del Vaticano, Biblioteca Apostolica Vaticana, Vat. Lat. 4IO4, f. 254 r). 
Hoc speculo spectans siquid in corpore pulchrj serva animo, turpis corrige siquid habes

Alius

Sola animum accendat Virtus: non gloria formae

20

[Paolo Panciatichi,] Al Re della fava, presentando un gambo di fava

Fonte: P, ff. 239v. A un Reame della Fava, che non sembra però aver nulla a che vedere con la Virtù, rimanda il capitolo Se giamai fu sententia promulgata (in lode del vino. Alli signori Giudici del Reame della Fava) di Giovanfrancesco Ferrari, cui segue una Risposta contro il Vino, a messer Hercole assertore della Fava (inc. Magnifico Signor, come fratello), in Le rime burlesche sopra varii, et piacevoli soggetti [...], In Venetia, Appresso gli Heredi di Marchiò Sessa, I 570 , cc. I $4 r$-I $8 r$. Una copia manoscritta dei due testi, probabilmente indipendente, è contenuta nel codice Capponiano i 52 della Biblioteca Apostolica Vaticana, ff. ı 04 -ı o6. Re di fava è comunque espressione che non comporta necessariamente un rituale accademico, perché rimanda semplicemente a una tradizione della notte dell'Epifania (viene acclamato re del convito chi trova una fava nella sua porzione di torta: GDLI XV 597-601, s.v. Fava').

Questa gentile et fruttifera pianta, da cui traete il nome altero et solo sotto suo augurio tua corona santa spande sua fama sopra 'l cielo a volo. Non più nespolo o melo oggi si vanta ma a la fava corre 'l mortal stuolo l'ama, et lo tien frutto superlativo quando si pianta in loco incarnativo.

Dunque vostra corona sacra et degna pigli tal pianta che fa tanto frutto la quale a noi mortal ne mostra e 'nsegna che per lei si mantiene, et nasce 'l tutto. Questa verace et gloriosa insegna facci fecondo ogni terreno asciutto ${ }^{108}$ et per che sia fruttifero et non reo si pianti in vigne et non nel Culiseo. 
* Ringrazio, al solito, Paolo Procaccioli per la cortesia e la pazienza con la quale viene incontro alle mie continue richieste. A Franco Pignatti devo inoltre alcune preziose indicazioni. La citazione del titolo è prelevata da una lettera di Paolo Giovio a Dionigi Atanagi, «Dal Museo a XXIIII di Gennaio MDXL» (PAolo Giovio, Lettere volgari di Mons. Paolo Giovio da Como Vescovo di Nocera. Raccolte per messer Lodovico Domenichi [...], Venezia, Giovan Battista et Marchion Sessa, I 560 , c. $8 \circ$ r).

I. Mi limito a rinviare a due saggi degli anni Ottanta che mi sono sempre parsi particolarmente convincenti: V. DE CAPRIO, Roma, in Letteratura italiana. Storia e geografia, II. L'età moderna, I, Torino, Einaudi, I988, pp. 327-472 (in particolare le pp. 453-63; dello stesso autore è importante anche I cenacoli umanistici, in Letteratura italiana, I. Il letterato e le istituzioni, Torino, Einaudi, I982, pp. 799-822); e D. RomeI, Roma I532-I537: accademia per burla e poesia "tolta in gioco" [1984], ora in Id., Da Leone X a Clemente VII. Scrittori toscani nella Roma dei papati medicei (ISI3I534), Manziana, Vecchiarelli, 2007, pp. 205-42. Per una bibliografia selecta (il documento è stato aggiornato il 24 marzo 2006) si può consultare la voce Burleschi immessa in rete dallo stesso Romei sul sito di Cinquecento plurale: http://www.nuovorinascimento.org/cinquecento/burleschi.pdf.

2. Romei, Roma I532-I53 cit., p. 2 IO.

3. De Caprio, Roma cit., pp. 453-63.

4. Mi riferisco per esempio a due saggi, veramente sessantottini, di G. Ferroni, Lettere e scritti burleschi di Annibal Caro tra il I532 e il I542, in «Palatino», XII (I968), pp. 374-86 e M. FEO, Dal pius agricola al villano empio e bestiale (a proposito di una infedeltà virgiliana del Caro), in «Maia», XX (1968), pp. 89-1 36 e 206-23; maestri che hanno dato poi il meglio di sé in altri ambiti di indagine.

5. Secondo la nota formula contenuta in una lettera di Annibal Caro ai familiari di Giovanni Gaddi, Castro, I 3 ottobre i 532 (Annibal Caro, Lettere familiari, edizione critica con introduzione e note a c. di A. Greco, Firenze, Felice Le Monnier, i 95 7-196i, vol. I, p. 5).

6. M.C. Figorilli, Meglio ignorante che dotto. L'elogio paradossale in prosa nel Cinquecento, Napoli, Liguori, 2007 , p. 2 (e anche: «Ma la scelta del gioco [...] non va interpretata come fuga nel disimpegno, come pretesto per un diletto separato, conclusus, e per ciò stesso momentaneo», p. 28). A questa interpretazione si è opposto decisamente in passato anche Antonio Sorella, come risulta evidente dal titolo stesso di una sua recente comunicazione: Letteratura burlesca e impegno intellettuale, in Annibal Caro a cinquecento anni dalla nascita. Atti del Convegno di studi, Macerata, I6-I 7 giugno 2007, a c. di D. Poli, L. Melosi, A. Bianchi, Macerata, Eum, 2009, pp. 73-105 (la data di pubblicazione non è molto significativa, perché il testo presenta riflessioni avviate almeno un decennio prima). Su Opsimathes si veda ora S. Adorni Braccesi, Tra ermetismo ed eresia: il Paradoxe contre les Lettres di Opsimathes (Lion I545-I546), in «Bruniana \& Campanelliana», XVII (200I), pp. 457-7I.

7. A. Corsaro, Il poeta e l'eretico. Francesco Berni e il 'Dialogo contra i poeti', Firenze, Le Lettere, 1988 . 
8. Romei, Roma I532-I537 cit., pp. 234, 237 e 24I. Una via intermedia tra le due precedenti sembra battere P. Cosentino, L'Accademia della Virtù: dicerie e cicalate di Annibal Caro e di altri virtuosi, in Cum notibusse et comentaribusse. L'esegesi parodistica e giocosa del Cinquecento. Seminario di Letteratura italiana, Viterbo, 23-24 novembre 2001, a c. di A. Corsaro e P. Procaccioli, Manziana, Vecchiarelli, 2002, pp. 177-92; Ead., Fra commento parodico e lettera faceta: Annibal Caro scrittore burlesco, Annibal Caro a cinquecento anni dalla nascita cit., pp. I I 5-38 (se ne legga in particolare la chiusa, pp. I 3 I-32).

9. Su Francesco Maria Molza (I 489-1 544) si dispone ora della recentissima voce di F. Pignatti sul Diz̨ionario biografico degli Italiani, Roma, Istituto dell'Enciclopedia italiana, vol. 75 [20I I], pp. 45 I-6I; cui anche si deve l'ampio e circostanziato saggio che compare in questo stesso fascicolo. Molto datata, invece, la voce dedicata al Bini da G. Ballistreri, Roma, Istituto dell'Enciclopedia italiana, vol. ı [1968], pp. 5 I0-I 3. Accenni recenti al Bini si riscontrano sparsamente nella letteratura sui cosiddetti berneschi, ma manca una sintesi aggiornata.

Io. Tra i quali occorrerà del resto fare un po' d'ordine. I due interventi 'barbari' pubblicati dall'Atanagi (Spirti nobili, che felicemente e $O$ come virtute ben posasi in altra colonna, in De le rime di diversi nobili poeti toscani [...], In Venetia, Appresso Lodovico Avanzo, i 565 , vol. II, c. 3 I $r-v$ ) afferiscono infatti certamente a una seconda fase della Virtù, sotto il patrocinio e l'egida di Francesco Colonna. L'ottava O Bella Dea, che da celesti giri, pure edita dall'Atanagi (De le rime di diversi nobili poeti toscani cit., vol. I, c. $43 v$ ), anche per la scelta del metro, sembra rimandare invece alla primissima fase del sodalizio.

i i. Claudio Tolomei, De le lettere [...] lib. sette, In Vinegia, Appresso Gabriel Giolito de' Ferrari, I 547, c. I 20 r.

I 2. Rigoglioso e non spregevole rimatore romano della celebre famiglia, autore anche di una commedia (Gli errori, Venezia, per Cornelio de Nicolini da Sabbio, a instantia de Marchiò Sessa, s.d., ma probabilmente postuma), morì verosimilmente il 25 settembre i 563 , secondo una nota apposta da Paolo Panciatichi a un suo epicedio, inedito: «Jacopo Cenci in questo luogo aprico | iace sepolto: poeta eccellente, | per sua virtù amato dalla gente, | fu ben roman, ma di costume antico» (Pistoia, Biblioteca Forteguerri, B I 76, f. $374 r$ ).

I 3. Sul Corvino, maestro di casa di Guido Ascanio Sforza di Santa Fiora, corrispondente dell'Aretino, celebre antiquario menzionato da Enea Vico come uno dei più importanti collezionisti romani, si vedano le notizie da me raccolte in E. Garaveldi, Stravaganze di Annibale. Parodie (amorose) cariane in verso e prosa, in Extravagances amoureuses: l'amour au-delà de la norme à la Renaissance. Stravaganze amorose: l'amore oltre la norma nel Rinascimento. Actes du colloque international du Groupe de recherche Cinquecento plurale, Tours, i 8-20 septembre 2008, sous la direction de É. Boillet et C. Lastraioli, Paris, Champion, 201 2, p. 228; cui si può aggiungere una testimonianza dell'Atanagi, che lo dice «gentilhuomo Romano, specchio già d'ogni gentile costume, et virtù in terra, et hora ornamento del cielo» (De le rime di diversi nobili poeti toscani cit., vol. I, c. Hh8r).

14. De le rime di diversi nobili poeti toscani cit., vol. I, c. $73 v$. 
I 5. Sull'Atanagi ( 1 504-1 573): C. Mutini, Atanagi, Dionigi, in Diz̧ionario biografico degli Italiani, vol. 4 [1962], pp. 503-506; G. МЕYRAт, Dionigi Atanagi e un esempio di petrarchismo nel Cinquecento, «Aevum», LII (i 978), pp. 450-58; A. Corsaro, Dionigi Atanagi e la silloge per Irene da Spilimbergo. Intorno alla formazione del giovane Tasso, «Italica», n. 75 (1998), pp. 4I-6 г; Figorilli, Meglio ignorante che dotto cit., pp. Iо9- I 3.

16. De le rime di diversi nobili poeti toscani cit., vol. I, c. Lliv.

17. C. DionisotTi, Tradizione classica e volgarizzamenti, in Id., Geografia e storia della Letteratura italiana, Torino, Einaudi, I967, p. I74.

i 8. E. Garavelli, Presenze burchiellesche (e altro) nel Commento di Ser Agresto di Annibal Caro, in «La fantasia fuor de' confini». Burchiello e dintorni a 550 anni dalla morte (1449-1999), Firenze, Palazzo Strozzi, 26 novembre i999, a c. di M. Zaccarello, Roma, Edizioni di Storia e Letteratura, 2002, pp. 195-239; «Perché Prisciano non facci ceffo». Ser Agresto commentatore, in Cum notibusse et comentaribusse cit., pp. 57-77; I pentimenti di Ser Agresto. Terza variazione sul Commento alla Ficheide di Annibal Caro, «Filologia e Critica», XXVIII (2003), pp. I 8 I-208. E si veda anche L. BALlone, L'enigma carnevalesco di Annibal Caro e una "ficata" del Valla, in Cum notibusse et comentaribusse cit., pp. 79-97. Non più che una scrollata di spalle merita invece il giudizio di chi non ha saputo vedere nel Ser Agresto altro che «paroliberismo senza logica e, in fondo, senza giustificazioni che non siano quelle d'un procedere comico o almeno ridevole».

19. [Annibal Caro], Commento di Ser Agresto da Ficaruolo sopra la prima ficata del Padre Siceo, [colophon: «In Baldacco, per Barbagrigia da Bengodi: | [...] Uscita fuora co' Fichi, | alla prima acqua d'Agosto. | l'Anno. | M.D.XXXIX.»], p. 7 (esemplare utilizzato: Firenze, Biblioteca Nazionale Centrale, Nencini F 7 I 37). «Zugo» vale 'minchione' (Grande Dizionario della Lingua italiana, dir. da S. Battaglia, Torino, Utet, I96r-2002 [d'ora innanzi GDLI], vol. XXI, 2003, p. I 107).

20. Così è ironicamente definita una certa Laura de' Mosti (che Menghini e Greco propongono di identificare con Laura Terracina, allora peraltro giovanissima) in una lettera del Caro al Molza del i 8 maggio i 58 (Annibal Caro, Lettere familiari cit., vol. I, pp. 97-98).

2 I. La prima (e unica) raccolta più o meno organica di testi dei Virtuosi fu tentata da Bartolomeo Gamba (Dicerie di Annibal Caro e di altri a' re della Virtù, CalveyHall [ma: Venezia, Alvisopoli], i 82 I). Il raro opuscolo contiene la Nasea e la Diceria di S. Nafissa del Caro, la Cotognata e il Bicchiere del Bini, le dicerie di Cincio, Gualtieri e Martirano e la lettera firmata da «Grassino Formaggiaro» che introduce la Formaggiata del Landi. Solo la Diceria di S. Nafissa era inedita a quella data (è curioso che continui a rispuntare l'equivoco di una princeps datata I 547 , ingenerato dalla menzione contenuta nella lettera del Bonfadio di cui sotto, nota 65 , pubblicata appunto in quell'anno). Gli altri testi sono esemplati dalle Lettere facete dell'Atanagi (Nasea, Cotognata, Cincio: De le lettere facete, et piacevoli di diversi grandi buomini, et chiari ingegni, raccolte per M. Dionigi Atanagi, libro primo, In Venetia, appresso Bolognino Zaltieri, i 56 I, pp. 33-84 e 298-300; uso l'esemplare segnato MD 400 VIII 23 della Biblioteca Nazionale [Kansalliskirjasto] di Helsinki) e del Turchi (Gualtieri e Martirano: Delle lettere facete, et piacevoli, di diversi grandi buomini, et 


\section{EnRico Garavelli}

chiari ingegni, scritte sopra diverse materie, raccolte per M. Francesco Turchi, libro secondo, In Venetia, [Aldo Manuzio?], I 575 , pp. 203-Io); il solo Bicchiere del Bini è prelevato dal Secondo libro dell'opere burlesche, cit., cc. I $22 v$ - I $25 r$. Un'esposizione critica dei testi raccolti dal Gamba ha tentato Cosentino, L'Accademia della Virtù cit. Sulle Lettere facete: Figorilli, Meglio ignorante che dotto cit., pp. I04-I 50 e L. Braida, Libri di lettere. Le raccolte epistolari del Cinquecento tra inquietudini religiose e "buon volgare", Roma-Bari, Laterza, 2009, pp. I 83-92. Piuttosto complessa è la storia della prima edizione Zaltieri, di cui esistono almeno due emissioni, con diversi frontespizi, dediche ritoccate ed estese ricomposizioni interne; non molto soccorre la nota premessa da Silvia Longhi alla ristampa anastatica del Primo libro (Bologna, Forni, I99I, pp. V-VII).

22. Sul collezionismo rinascimentale mi limito a rimandare a C. Franzoni, "Rimembranze d'infinite cose». Le collezioni rinascimentali di antichità, in Memoria dell'antico nell'arte italiana, a c. di S. Settis, I. L'uso dei classici, Torino, Einaudi, I 984 , pp. 299-360. Del coinvolgimento del Gaddi non occorre dire; quanto al Pio, basti ricordare che ne era segretario l'aretino Bernardo Boccarini, che contribuì alla Nuova poesia con cinque componimenti (Versi et regole della nuova poesia toscana. In Roma per Antonio Blado d'Asola. I539, edizione e introduzione di M. Mancini, Manziana, Vecchiarelli, I 996, cc. O2 $r$-3v). Quanto alla partecipazione del Caro a quella stagione di entusiasmi per le discipline antiquarie, mi limito a rimandare agli spunti proposti nel mio Stravaganze di Annibale cit., pp. 226-33.

23. I. Foschi - G. Rebecchini, Medici, Ippolito de', in Dizionario biografico degli Italiani, vol. 73 [2009], pp. 99-103.

24. Lettera a Sigismondo d'Este, Roma, i 8 luglio i54I (che leggo in LuCA Contile, Delle lettere [...], Pavia, Girolamo Bartoli, i 564, pp. i9-20).

25. Tra l'altro, si utilizzano i due termini tecnici di «animo» (anima) e «corpo» che saranno canonizzati molti anni più tardi dal Giovio e dal Ruscelli (G. Arbizzoni, «Un nodo di parole e di cose». Storia e fortuna delle imprese, Roma, Salerno, 2002, pp. I I-36).

26. Tutte le citazioni dalla Cotognata sono tratte da De le lettere facete, et piacevoli [...] libro primo cit., pp. $270-78$.

27. Pietro Bembo, Prose della volgar lingua. Gli Asolani. Rime, a c. di C. Dionisotti, Torino, Utet, I966, pp. 89-Ios (I SS VIII-XI). Per il Bembo provenzalista è d'obbligo il rimando a S. DeBenedeTti, Gli studi provenzali in Italia nel Cinquecento, Torino, Loescher, I9I I, ad indicem.

28. Giuseppe Cincio, medico di Margherita d'Austria, fu Chierico del Sacro Collegio almeno nel i 54I e nel i 544. Sulla sua diceria si veda R. Gigliucci, Breve dolcezza, lungo malanno. Parodie dei contrapposti petrarcheschi, in Cum notibusse et comentaribusse cit., pp. 199-206 (in particolare pp. 199-200).

29. Pietro Paolo Gualtieri, [La corona di gramigna e una terzina del Petrarca $=$ TC III i 8 i: Come ne l'ossa il suo fuoco coperto] e Coriolano Martirano, Amore fa gli uomini balordi [su TC III 175: So, come Amor saetta, e come vola] (si veda supra, nota 2 I). Sul Gualtieri (I 5OI-I 572), aretino, scriptor della Cancelleria apostolica e 
poi segretario ai brevi, stretto collaboratore di Marcello Cervini durante il suo brevissimo pontificato, si veda la voce di V. Gallo sul Dizionario biografico degli Italiani, vol. 60 [2003], pp. 217-I8. Partecipò tra l'altro alla raccolta dei Versi et regole della nuova poesia toscana con poco meno che una ventina di componimenti (cc. $\mathrm{E}_{3} r-4 v, \mathrm{O}_{2} r, \mathrm{~T}_{4} v$-T6r). Il cosentino Martirano (I 503-I 557), a quei tempi vescovo di San Marco in Argentano, avrà un ruolo di rilievo durante le prime fasi del Concilio e diventerà nel I 554 segretario del Regno di Napoli (si veda la voce di E. VAleri sul Dizionario biografico degli Italiani, vol. 7I [2008], pp. 34I-44).

30. Lettera al Varchi, Roma, io gennaio I 538 (AnNIBAL CARO, Lettere familiari cit., vol. I, p. 59).

3 I. Per Giovanni Gaddi mi limito a rimandare alla voce di V. Arrighi sul Dižionario biografico degli Italiani, vol. 5 I [1998], pp. I 56-58 (qualche integrazione in Garavelli, Stravaganze di Annibale cit., pp. 226-27).

32. Per quanto riguarda la datazione della Diceria di S. Nafissa, si badi che: il Caro la cita nel Proemio del Commento di Ser Agresto (Annibal Caro, Commento di Ser Agresto cit., c. 4r), sicché è anteriore almeno all'agosto i 539; nella redazione autografa della lettera al Varchi del 10 marzo i 538 il Caro parla delle sue "pappolate», al plurale: cioè appunto almeno della Diceria di S. Nafissa e della Nasea (nella redazione riveduta del Fonds Italien 1707 della Bibliothèque Nationale di Parigi Annibale riduce l'accenno, chiarendolo, alla sola Nasea, certo per lo stesso intento autocensorio che gli consigliò di non pubblicare l'altra diceria insieme con il Commento di Ser Agresto: Annibal Caro, Lettere familiari cit., vol. I, pp. 73 e 70); che il destinatario della S. Nafissa sia Giovanni Gaddi non è dubbio, benché non siano mancate in passato altre proposte, favorite anche dall'erroneo sottotitolo di Diceria al Sesto Re della Virtù presente in una parte consistente della tradizione manoscritta (GARAveldi, Stravaganze di Annibale cit., pp. 220-33, con bibliografia pregressa).

33. «CARTE: Commendava forte gl'inventori di qualche bel giuoco, come quel trovato dal signor Claudio Tolomei che si cognomina la Virtù. Padovano: Si dice che egli è di sessanta carte. CARTE: Il suo è proprio un giocar da vertuosi e degno d'essere uscito da un tanto intelletto» (Pietro Aretino, Le carte parlanti, a c. di G. Casalegno e G. Giaccone, Palermo, Sellerio, I992, pp. 3 I I-I 2). Meglio s'intende, dunque, il cenno del Caro al Leoni: «Ill Regno de la Virtù è in declinazione, e la primiera, se non si rimette, gli darà scacco matto» (lettera a G.F. Leoni, Roma, io aprile i 538 , in Annibal Caro, Lettere familiari cit., vol. I, p. 83).

34. Lettera al Varchi, Roma, io marzo i 538 (Annibal Caro, Lettere familiari cit., vol. I, p. 72).

35. Cui infatti si allude già in una lettera al Molza, Napoli, io maggio i 538 (Annibal Caro, Lettere familiari cit., vol. I, p. 9I).

36. Il secondo libro dell'opere burlesche cit., c. 7I $r$. Se così fosse, questa quarta serata sarebbe stata realizzata dopo il 24 gennaio, data dell'inizio del viaggio del Franzesi (la si ricava dalla commendatizia scritta dal Caro a Paolo Manuzio in quella data: Annibal Caro, Lettere familiari cit., vol. I, p. 6i). 
37. Sul Leoni, si veda almeno P. Cosentino, Leoni, Giovan Francesco, in Dizionario biografico degli Italiani, vol. 64 [2005], pp. 591-92.

38. «La mia fagiolata non uscirà fuori che non eschi delle man vostre» (lettera al Varchi, Roma, io marzo i 538: Annibal Caro, Lettere familiari cit., vol. I, p. 72).

39. Lettere al Maffei, Napoli, io aprile i 538 , e al Leoni, Roma, io aprile I 538 (Annibal Caro, Lettere familiari cit., vol. I, pp. 79 e 83).

40. Vedi supra, nota I 3 .

4I. Per questa ipotesi rimando al cappello introduttivo al testo n. 9, alquanto problematico. A un «Re Cucullato» il Caro allude nella lettera al Leoni del io aprile i 538 (Annibal Caro, Lettere familiari cit., vol. I, p. 8I). Si tratta sicuramente dello stesso «Padre Cucullato» citato nella Diceria di S. Nafissa (Annibal Caro, Gli straccioni commedia. La Ficheide comento. La Nasea e La statua della Foia cit., p. I 85 ). Ora, dal momento che le proposte interpretative avanzate dalle auctoritates accademiche (più o meno travestite) sono citate in quel testo sequenzialmente e senza ripetizioni, non è possibile identificare questo personaggio con gli altri menzionati prima e dopo. Ne consegue che il Padre cucullato non può essere: Bini, Sebastiano del Piombo, Molza, Corvino, Porrino, Silvestro Battiloro, Tolomei, Giovanni Battista Galletti, Ferdinando Balamio, Cincio, Leoni (né Caro, autore, né Gaddi, destinatario del testo). L'unico sicuro re che non compare in questa lista è Paolo Panciatichi, che però non risulta fosse frate. Mi chiedo se il suo alter ego poetico del Palandrano potesse tollerare la sostituzione burlesca di Cocolla. Altri ha pensato in passato a Giovanni Della Casa (sulla cui partecipazione alla Virtù, da ultimo, A. Sorella, Un poemetto inedito attribuibile a Della Casa, in Giovanni Della Casa ecclesiastico e scrittore. Atti del convegno, Firenze-Borgo San Lorenzo, 20-22 novembre 2003, a c. di S. Carrai, Roma, Edizioni di Storia e Letteratura, 2007, pp. 259-280). Una mia precedente ipotesi (Ludovico Fabri DA FAnO, in Stravaganze di Annibale cit., p. 224) continua a rimanere in verità irrelata.

42. In mancanza di una voce specifica sul Dizionario biografico degli Italiani, mi limito a ricordare che il Colonna, nipote di Pompeo (e dedicatario della vita di quest'ultimo scritta da Paolo Giovio e volgarizzata da Lodovico Domenichi) e zio di Marcantonio, fu dal i 529 abate commendatario di Subiaco, dal I 534 al I 544 arcivescovo di Rossano e dal I 544 alla morte ( 560 ) vescovo di Taranto.

43. De le rime di diversi nobili poeti toscani cit., vol. II, c. 3 I $r$-v. È del tutto priva di fondamento la notizia secondo la quale il Tolomei avrebbe riunito l'accademia in casa propria dopo la morte del Colonna, per il semplice fatto che il Tolomei gli premorì.

44. Questo significa «risentimento della Virtù», non 'ira', come inteso dal Greco e da altri (si legga la lettera al Leoni da Forlì, 20 maggio i 540, in AnNibal Caro, Lettere familiari cit., vol. I, pp. I94-95).

45. Due orazioni in lingua toscana. Accusa contra Leon Secretario, di secreti rivelati. Difesa, In Parma, appresso Sette Viotto, I 547. 
46. Cui già vengono assegnate quindici anni più tardi nella raccolta curata da Francesco Sansovino, da cui, per necessità, sono costretto a citare (Diverse orationi volgarmente scritte da molti huomini illustri de' tempi nostri [...] raccolte, rivedute et corrette per Francesco Sansovino, In Venetia, appresso Fran. Sansovino, I 56I, cc. 59r-68r).

47. Figorilli, Meglio ignorante che dotto cit., p. 7, nota 9 ricorda precedenti umanistici e soprattutto il «procedimento palinodico» che si rileva «alla radice dello schema doppio adottato da Ortensio Lando in alcune sue opere».

48. In alternativa, ma mi sembra improbabile, si potrebbe pensare alla primavera del I 543 , se si dovesse leggere un accenno alla diatriba in questo passaggio, non però del tutto perspicuo, della lettera che Tolomei indirizza al Leoni da Roma il 29 giugno di quell'anno: «M'è piaciuto il vostro ridurvi a penitenza, onde io come buon christiano vi perdono; ma guardate di non tornare subbito al nuovo peccato, di che m'avvedrò ben tosto, e vel ricordarò rigorosamente» (CLAudio ToloMEI, De le lettere [...] lib. sette cit., c. I $26 r-v)$. Un mese dopo, il 20 luglio, sempre il Tolomei scrive al Leoni (a Padova con Ranuccio Farnese): «Tutta la Virtù vi ricorda, vi disidera, vi brama ardentemente» (Claudio Tolomei, De le lettere [...] lib. sette cit., cc. 55v-56r).

49. La si può vedere nella scheda di EDITI6 on line:

http://edit 6 6.iccu.sbn.it/web-iccu/imain.htm.

50. Sul Landi, si veda la recente voce di P. Cosentino, Landi, Giulio, in Dizionario biografico degli Italiani, vol. 63 [2004], pp. $385-89$.

5 I. Sul Bonfadio, oltre alla brevissima e ormai datata voce di R. URBAni, Bonfadio, Iacopo, in Dizionario biografico degli Italiani, vol. I 2 [1970], pp. 6-7, rimando almeno agli atti del seminario Jacopo Bonfadio a cinquecento anni dalla nascita. Atti del Convegno, Roè Volciano, 25 ottobre 2008, a c. di A. Bonomi e S. Zaboni, Roè Volciano, Comune di Roè Volciano, 2009 (consultabili anche on line:

http://www.jacopobonfadio.it/pdf/volume-atti-definitivo.pdf).

52. D. E. Rhodes, Accertamenti tipografici sulla «Formaggiata» del conte Giulio Landi, I542, «Bollettino storico piacentino», LXXX (1 985 ), pp. 70-73, e M. BAUCIA, Accertamenti storico-letterari sulla «Formaggiata» del Conte Giulio Landi, I542, «Bollettino Storico Piacentino», LXXXI (1986), pp. I04-2 I. Si veda anche Figorilli, Meglio ignorante che dotto cit., pp. I I 8-22.

53. Claudio Tolomei, De le lettere [...] lib. sette cit., c. i i $7 v$ («Del formaggio che m'inviate, vi ringrazio assai, ma molto più vi ringraziarò, quando sarà arrivato, $\mathrm{e}$ molto più senza dubbio, quando io lo mangiarò, e lo trovarò buono, sì come lo spero, venendo da coteste parti, e da voi»).

54. Ai riferimenti «come il naso di messer Francesco d'Ancona è Re de gli altri nasi, approvato da una cima di galante huomo», c. $9 v$ e "quel che fece il naso dell'anconitano famoso», c. $17 r$ [recte $2 \mathrm{I} r$ ], già individuati da Baucia (Accertamenti storico-letterari cit., p. I I 2 nota 34) si può aggiungere il gioco sul naso del Re di Francia, contrapposto alla bocca di Carlo V («ha il naso grande come il Re di Francia; la bocca non è totalmente da Imperadore, ma da Re sì bene», c. I 7v), da 
confrontare con «Sopra che si fonda l'oppenione d'un mio compagno: quale è, che Carlo V sia hoggi sì grande Imperadore, perchè si truova sì gran Bocca: et che Francesco Re di Francia sia sì gran Re, perchè ha sì gran Naso» (cito dalla princeps del i 539: Annibal Caro, Commento di Ser Agresto cit., p. 70).

55. Dopo aver ricordato con nostalgia $i$ tempi in cui «insieme adoravamo quel nostro terreno Idolo» (cioè, appunto, Ippolito), Tolomei aggiunge: «Hora tra que’ tempi e questi è passato un grande spazio di tenebre, e un longo intervallo di silenzio tra noi», nemmeno interrotto dalla corrispondenza epistolare (infatti, «il voler senza occasione intrattenerci con lettere, non era altro ch'una cerimonia piena di vanità e di vento»). Finalmente adesso ha saputo qualcosa del vecchio amico da Luca Contile, appena rientrato a Roma (Claudio Tolomei, De le lettere [...] lib. sette cit., c. $72 r-v)$.

56. Lettera al Varchi, Roma, io marzo i 538 (Annibal Caro, Lettere familiari cit., vol. I, p. 72).

57. Nella citata voce Landi del Diz̧ionario biografico degli Italiani, Paola Cosentino riprende la vecchia proposta del settecentista piacentino Cristoforo Poggiali (C. Poggiali, Memorie per la storia letteraria di Piacenza, vol. II, Piacenza, Orcesi, I789, p. 199) e identifica il destinatario della Formaggiata in Claudio Tolomei; ma già Baucia ha fatto notare che il Tolomei è citato come persona differente dal destinatario a c. I $_{7} r$ [recte 2 I $\left.r\right]$ : «era veramente impresa per quei divini, et nella virtù baroni, et principi, et di vostra Maestà servitori affezionatissimi; messer Claudio, il Molza, messer Bino [...]» (Baucia, Accertamenti storico-letterari cit., p. I I 3).

58. Lettera a Silvestro da Prato, Velletri, 30 aprile i 538 (Annibal Caro, Lettere familiari cit., vol. I, pp. 83-89).

59. Annibal Caro, Gli straccioni commedia. La Ficheide comento. La Nasea e La statua della Foia cit., p. I 83.

6o. Non ricavo nulla nemmeno dal recente volume di A. Nuovo-C. Coppens, I Giolito e la stampa nell'Italia del XVI secolo, Genève, Droz, 2005: il Giolito, che certamente stampò il volume a spese del Landi, non si curò di chiederne il privilegio.

6r. P. Procaccioli, Cinquecento capriccioso e irregolare. Dei lettori di Luciano e di Erasmo; di Aretino e Doni; di altri peregrini ingegni, in Cinquecento capriccioso e irregolare. Eresie letterarie nell'Italia del Classicismo. Seminario di Letteratura italiana (Viterbo, 6 febbraio I 998), a c. di Id. e A. Romano, Manziana, Vecchiarelli, I999, pp. 23-24. Il rilievo è già in BAUCIA, Accertamenti storico-letterari cit., pp. I I o- I I, che ne deduce un argomento valido per distinguere autore del testo e autore della lettera ai lettori.

62. Il caso più eclatante è la citazione della Formaggiata nella Libraria come opera offerta «all'immortal cardinale de Medici»; che sembra più un lapsus di lettore distratto che la dimenticanza di un editore iperattivo (ANTOnFrancesco Doni, La libraria, a c. di V. Bramanti, Milano, Longanesi, I972, pp. I 20-2 I).

63. IAcopo Bonfadio, Le lettere e una scrittura burlesca, testo con introduzione e commento di A. Greco, Roma, Bonacci, 1978, pp. I 57-67. In precedenza: De le 
lettere facete, et piacevoli [...] libro primo cit., pp. 323-36. Uno squarcio anche in P. Fanfani, Paolo Panciatichi, «Il Piovano Arlotto. Capricci mensuali di una brigata di begliumori», I (1 858 ), pp. 209-10.

64. Dalla menzione del Bronzino ricaviamo che dovesse essere morto ante i 559 (Agnolo di Cosimo [il Bronzino], I salterelli dell'Abbrucia sopra i Mattaccini di Ser Fedocco, a c. di C. Rossi Bellotto, Roma, Salerno Editrice, I998, pp. 27-28 e 83; la Rossi ipotizza che il suo cognome fosse Cambi). Si veda anche M. Plaisance, L'Accademia e il suo Principe. Cultura e politica a Firenze al tempo di Cosimo I e di Francesco de' Medici, Manziana, Vecchiarelli, 2004, pp. I $50-5$ I e nota 76.

65. Iacopo Bonfadio, Le lettere e una scrittura burlesca cit., p. I 28.

66. Ma merita di essere ricordato il fatto che nella diceria si cita un componimento del Molza conservato a quanto pare nel solo codice Borgiano 367 , autografo (F. Baiocchi, Sulle poesie latine di Francesco M. Molza, Pisa, Nistri, I904, p. 82).

67. IAcopo Bonfadio, Le lettere e una scrittura burlesca cit., p. I 37.

68. Alcuni suoi testi furono pubblicati da Pietro Fanfani nel citato articolo apparso sul «Piovano Arlotto», I ( I 8 5 8), pp. 208-2 I: cinque sonetti caudati più altri frammenti. Due altri sonetti caudati (in realtà uno era già stato pubblicato dal Fanfani) furono proposti da Ottaviano Targioni Tozzetti in un opuscolo per Nozze Marradi-Foraboschi, 29 luglio i 883 (Firenze, Biblioteca Nazionale Centrale, Misc. I 83. 22). Lo stesso Targioni Tozzetti, in collaborazione con Ettore Tocci, avrebbe dovuto curare un'edizione antologica delle rime del Panciatichi per l'editore Vigo di Livorno; dell'opera si diede l'annuncio sul «Giornale Storico della Letteratura italiana», III (I 884), p. I 59, ma non se ne fece niente. Di altro non ho notizia; ma ricordo che nella seconda edizione del primo libro delle Lettere facete (Venezia I 582, pp. 223-34) gli venne attribuita la Furfanteria del Bonfadio (a tale attribuzione dà ancora credito F. SAvI, Paolo Panciatichi rimatore pistoiese del secolo $X V I$, «Bullettino Storico Pistoiese», LXXIV, s. VII, I972, pp. I23-27, a p. I26; all'articolo di Savi, che scriveva prima dell'edizione Greco delle Lettere del Bonfadio, faccio riferimento per le notizie seguenti).

69. Si tratta del manoscritto segnato D 3 I 3 della Biblioteca Forteguerri di Pistoia, noto come "codice Tonti"; sul quale si veda C. Rossi, Il Pistoia spirito bizzarro del Quattrocento, Alessandria, Edizioni dell'Orso, 2008, p. 79.

70. Lettere del Molza a Camillo Molza e a Gandolfo Porrino, s.d. [ma probabilmente Fondi, I 534 ] e Fondi, s.d. [ma ante io agosto i 535 ], in Francesco Maria Molza, Delle poesie volgari e latine cit., vol. I, pp. 57 e I 48 .

7 I. Firenze, Archivio di Stato, Mediceo del Principato, 4I 5, f. 838 e Firenze, Biblioteca Medicea Laurenziana, Archivio Buonarroti, V. N-R, ad vocem.

72. L. Dorez, La Cour du Pape Paul III d'aprés les registres de la trésorerie secrète (collection F. De Navenne), préface de P. de Nolhac, I. La Cour Pontificale, Paris, Librairie Ernest Leroux, 1932, p. 28 I e II. Les dépenses privées, p. I 2. 


\section{EnRico Garavelli}

73. De le rime di diversi nobili poeti toscani cit., vol. I, c. I $82 r$.

74. Anton Francesco Raineri, Cento sonetti, a c. di R. Sodano, Torino, Res, 2004, pp. 37-38 (si veda anche la breve scheda biografica approntata dalla curatrice a p. 299).

75. Nè più chiara luce ti poteva accendere Amore (Versi et regole della nuova poesia toscana cit., c. T2r).

76. Si legga la lettera che quegli gli indirizzò da Padova il 22 dicembre I 53 I (Pietro Bembo, Delle lettere [...] terzo volume, In Vinegia, [appresso Gualtero Scotto], I552, pp. 318-I9).

77. In particolare, da una lettera da Roma, i marzo i 539 veniamo a sapere che messer Claudio aveva provveduto a inviargli versi "barbari" già molti mesi prima della stampa del volume, che il colophon dice licenziato solo in ottobre (CLAudio Tolomei, De le lettere [...] lib. sette cit., c. I42v).

78. J. Delumeau, Une confrérie romaine au XVT siècle: l' Arciconfraternita del SS.mo Crocefisso in S. Marcello, "Mélanges d'archéologie et d'histoire», LXIII (I95I), p. 293.

79. Annibal Caro, Lettere familiari cit., vol. I, p. 23.

8०. G. Brunelui, Marcello II, papa, in Dizionario biografico degli Italiani, vol. 69 [2007], pp. 502-Io. Si veda anche il recentissimo C. Quaranta, Marcello II Cervini (I50II555). Riforma della Chiesa, Concilio, Inquisizione, Bologna, Il Mulino, 20 I I.

8i. Annibal Caro, Lettere familiari cit., vol. I, p. 24. Secondo un antico biografo, il Caro avrebbe addirittura pronosticato il cardinalato al Cervini (Pietro Polidori, De vita, gestis, et moribus Marcelli II Pontificis Maximi commentarius, Romae, Ex Typographia Hieronymi Mainardi, i744, p. Io).

82. Benedetto Varchi, Lettere I535-I562, a c. di V. Bramanti, Roma, Edizioni di Storia e Letteratura, 2008, p. 62. Due lettere del Cervini al Varchi, Roma, i9 febbraio e 29 dicembre I 536 sono pubblicate in V. Vianello, Il letterato, l'accademia, il libro. Contributi sulla cultura veneta del Cinquecento, Padova, Antenore, I 988 ,

p. $175-176$.

83. «tutti» BG.

84. Corretto su «parte».

85. Corretto su «fuoco».

86. «N’ognun» BG.

87. «Pastinaca», dal gr. $\delta \varkappa^{\prime} \varkappa \varsigma_{5}$, attraverso il latino daucus (GDLI IV i966, p. 44).

88. 'Clistere'. 
89. Cioè 'rame'.

90. G. Alberigo, Archinto, Filippo, in Dizionario biografico degli Italiani, vol. 3 [I96 I], pp. $76 \mathrm{I}-64$.

9I. Cioè di lentischio, con proprietà purgative, da mastichinus, calco di $\mu \alpha \sigma \tau i$ «เvos, da $\mu \alpha \sigma \tau i \zeta$, 'resina di lentischio' (GDLI IX 1975, p. 907).

92. 'Mestruo' (voce furbesca, GDLI IX 1975, pp. 77 I-2). Lo scambio omonimico è già nel Berni (Francesco Berni, Rime, a c. di D. Romei, Milano, Mursia, i 985 , p. 97).

93. Paolo III.

94. Corretto su «Corbo».

95. «Chiuso» aggiunto in interlinea.

96. «che» BG.

97. Univerbo (BG ha «Barba rossa»). Si tratta ovviamente del pirata Chairiddin Barbarossa (sull'impresa, si parta almeno da A. Kohler, Carlo V, Roma, Salerno Editrice, 2005, pp. 250-57).

98. Cioè Compartii, I pers. plurale del pass. remoto. Per la forma, si vedano almeno G. RoHLFs, Grammatica storica della lingua italiana e dei suoi dialetti. Morfologia, trad. di T. Franceschi, Torino, Einaudi, i 968, p. 3 i 8 [S 571] e Lodovico CASTELVETRO, Giunta fatta al ragionamento degli articoli et de' verbi di Messer Pietro Bembo, a c. di M. Motolese, Roma-Padova, Antenore, 2004, pp. I4I e I 46.

99. «Glaudio» è naturalmente Claudio Tolomei. Difficile invece individuare univocamente il «Gallo» in questione. La candidatura più probabile sembra quella di Giulio Gallo, al quale il Leoni indirizza il sonetto Gallo, ch'al patrio sen mesto ten vai (De le rime di diversi nobili poeti toscani cit., vol. II, c. $234 v$; nella stessa carta figura anche Quanto più lasso, il mio desir affreno). L'Atanagi lo descrive come «Giulio Gallo, gentilhuomo Romano, veramente gentile, et cortese, per vivezza d'ingegno, et per prontezza, et argutia di ragionare di raro valore, et di dolcissimo

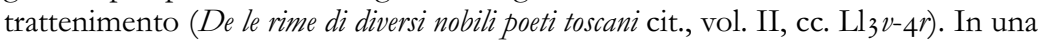
lettera ad Alessandro Corvino da Padova, 3 maggio I 549, si dichiara trentaseienne e cita un «M. Leone» (probabilmente il Leoni) e il «Bino» (cioè il Bini). Nell'epistolario cariano il Gallo figura ripetutamente: se ne deduce che fu per un certo tempo al servizio di Ottavio Farnese e più tardi, negli anni Sessanta, del cardinal Girolamo da Correggio. In alternativa, si potrebbe pensare all'urbinate Antonio Galli, più tardi corrispondente del Caro (E. Del Gallo, Galli, Antonio, in Dizionario biografico degli Italiani, vol. 51, I998, pp. 602-03). Un terzo Gallo, Giacomo, manda le Elegie del Molza, avute da Trifone Benci, al cardinal Alessandro Farnese con una lettera da Roma il 3 settembre i 544 (Lettere d'uomini illustri conservate in Parma nel R. Archivio dello Stato, [a c. di A. Ronchini], Parma, Dalla Reale Tipografia, I 853 , vol. I, pp. 98-9); ed è citato in una lettera del Caro a Girolamo Soperchio, Roma, 23 aprile i 548 (Annibal Caro, Lettere familiari cit., vol. II, p. 6o). 


\section{Enrico Garavelli}

ıо०. Corretto su «maestade».

I I. «che» BG.

I02. Come ricorda G.L. Beccaria, Sicuterat. Il latino di chi non lo sa: Bibbia e liturgia nell' italiano e nei dialetti, Milano, Garzanti, 200 I ${ }^{2}$, p. 62, San Piero per 'membro virile' (ovvio sviluppo della metafora delle chiavi) è attestato almeno nel Berni e nel Dolce. Si veda anche la nota 38 di Michel Plaisance al Piangirida del Lasca in Ludi esegetici (Berni, Comento alla Primiera - Lasca, Piangirida e Comento di maestro Niccodemo sopra il Capitolo della salsiccia), testi proposti da D. Romei, M. Plaisance, F. Pignatti, con una premessa di P. Procaccioli, Manziana, Vecchiarelli, 2005, p. I I 5 . Quanto a «farda», soccorre subito la prima Crusca: «catarro grosso, che si sputa nello spurgarsi, o sporcizia simile» (Vocabolario degli Accademici della Crusca, In Venetia, Appresso Giovanni Alberti, I6 г 2, s.v. Infardare [ho consultato l'edizione digitalizzata alla pagina http://vocabolario.signum.sns.it/]. Si veda anche GDLI V i 968, p. 660.

ı०3. «In» aggiunto in interlinea.

I04. «Inter vos» annotato sopra un «potitur» cassato.

I05. «Usque» («us3») corretto su «usj».

ı 6. Qui il copista aveva erroneamente anticipato il v. ıo, che ha successivamente cassato.

I07. «Vasi» aggiunto in interlinea.

I08. A «fecondo» segue l'erroneo e dittografico «ogni fecondo», depennato. 